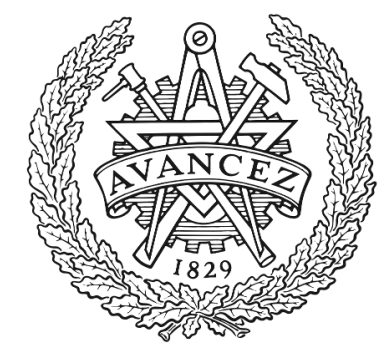

\title{
CHALMERS
}

UNIVERSITY OF TECHNOLOGY

\section{Quantum bits with Josephson junctions}

Downloaded from: https://research.chalmers.se, 2023-04-25 23:21 UTC

Citation for the original published paper (version of record):

Wendin, G., Shumeiko, V. (2007). Quantum bits with Josephson junctions. Low Temperature Physics, 33. http://dx.doi.org/10.1063/1.2780165

N.B. When citing this work, cite the original published paper. 


\title{
Quantum bits with Josephson junctions (Review Article)
}

\author{
G. Wendin and V. S. Shumeiko ${ }^{a)}$
}

Department of Microtechnology and Nanoscience, Chalmers University of Technology, Gothenburg, Sweden (Submitted January 15, 2007)

Fiz. Nizk. Temp. 33, 957-981 (September 2007)

\begin{abstract}
Recent demonstrations of macroscopic quantum coherence in Josephson junction based electronic circuits have opened an entirely new dimension for research and applications in the established field of Josephson electronics. In this article we discuss basic Josephson circuits for qubit applications, methods of quantum description of these circuits, and circuit solutions for qubit couplings. Principles of manipulation and readout of superconducting qubits are reviewed and illustrated with recent experiments using various qubit types. (C) 2007 American Institute of Physics.
\end{abstract}

[DOI: $10.1063 / 1.2780165]$

\section{INTRODUCTION}

Practical implementation of quantum computation algorithms ${ }^{1,2}$ requires the development of a special kind of hardware, which can broadly be described as a controllable many-body quantum network. The subject of this articlesuperconducting electrical circuits containing Josephson junctions-may serve as the elementary blocks of such a network: quantum bits. The possibility of achieving quantum coherence in macroscopic Josephson junction (JJ) circuits, envisioned by Leggett in the early $1980 \mathrm{~s},{ }^{3-5}$ came to reality almost 20 years later in the experimental demonstration of coherent quantum oscillation in a single Cooper pair box by Nakamura et al. $^{6}$ It would be fair to say that this breakthrough experiment represents the "tip of the iceberg": it rests on a huge volume of advanced research on Josephson junctions and circuits developed during the last 25 years. Some of this work has concerned fundamental research on Josephson junctions and superconducting quantum interferometers (SQUIDs), aimed at understanding macroscopic quantum tunneling (MQT) ${ }^{7-9}$ and macroscopic quantum coherence $(\mathrm{MQC}),{ }^{5,10}$ providing the foundation of the persistent current flux qubit ${ }^{11-13}$ and the JJ phase qubit. ${ }^{14-16}$ However, there has also been intense research aimed at developing superconducting flux-based digital electronics and computers. Moreover, in the 1990s, based on the Coulomb blockade theory of Josephson tunneling, ${ }^{17,18}$ the single Cooper pair box (SCB) was developed experimentally ${ }^{19,20}$ and used to demonstrate the quantization of Cooper pairs on a small superconducting island, which is the foundation of the charge qubit. ${ }^{6,21}$

Since then there has been a steady development, ${ }^{22-26}$ with observation of microwave-induced Rabi oscillation of the two-level populations in charge ${ }^{27-29}$ and flux ${ }^{30-33}$ qubits and dc-pulse-driven oscillation of charge qubits with rf-SET detection. ${ }^{34}$ An important step is the development of the charge-phase qubit, a hybrid version of the charge qubit consisting of an SCB in a superconducting loop, ${ }^{27,28}$ demonstrating Rabi oscillations with very long coherence time, of the order of $1 \mu \mathrm{s}$, allowing a large set of basic and advanced ("NMR-like") one-qubit operations (gates) to be performed. $^{29}$ In addition, coherent oscillations have been demonstrated in the "simplest" JJ qubits of them all, namely a single Josephson junction, ${ }^{14,15,35,36}$ or a two-JJ dc SQUID, ${ }^{16}$ where the qubit is formed by the two lowest states in the periodic potential of the JJ itself.

Although a powerful JJ-based quantum computer with hundreds of qubits remains a distant goal, systems with 5-10 qubits will be built and tested by, say, 2010. Pairwise coupling of qubits for two-qubit gate operations is then an essential task, and a few experiments with coupled JJ-qubits with fixed capacitive or inductive couplings have been reported, ${ }^{37-42}$ in particular the first realization of a controlled-NOT gate with two coupled $\mathrm{SCBs},{ }^{38}$ used together with a one-qubit Hadamard gate to generate an entangled two-qubit state.

For scalability and simple operation, the ability to control qubit couplings, e.g., switching them on and off, will be essential. So far, experiments on coupled JJ qubits have been performed without direct physical control of the qubit coupling, but there are many proposed schemes for two(multi)qubit gates based on fixed or controllable physical qubitqubit couplings or tunings of qubits and bus resonators.

This article aims at describing the inner workings of superconducting JJ circuits, how these can form two-level systems acting as qubits, and how they can be coupled together to multi-qubit networks. Since the field of experimental qubit applications is only five years old, it is not even clear whether the field represents an emerging technology for computers. Nevertheless, the JJ-technology is presently the only example of a working solid state qubit with long coherence time, with demonstrated two-qubit gate operation and readout, and with potential for scalability. This makes it worthwhile to describe this system in some detail.

It needs to be said, however, that much of the basic theory for coupled JJ-qubits was worked out well ahead of experiment, ${ }^{21,43,44}$ defining and elaborating the basic operation and coupling schemes. Several reviews on the subject are currently available, ${ }^{24,25,44-46}$ which describe the basic principles of a multi-JJ-qubit information processor, including essential schemes for qubit-qubit coupling. The ambition of the present article is to provide a both introductory and in-depth overview of essential Josephson junction quantum circuits, discuss basic issues of readout and measurement, and connect to the recent experimental progress with JJbased qubits for quantum information processing. 


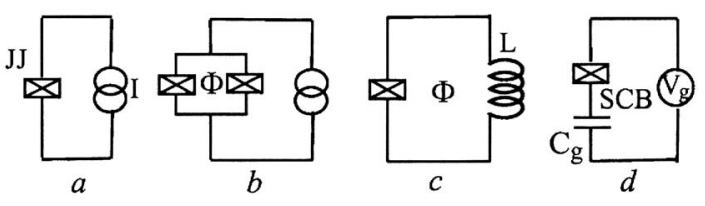

FIG. 1. Basic superconducting circuits for qubit applications: current-biased Josephson junction $(a)$; dc SQUID (b); rf SQUID (c); single Cooper pair box $(d)$; the crossed box indicates a combination of a Josephson tunneling element and a junction capacitor connected in parallel.

\section{QUANTUM SUPERCONDUCTING CIRCUITS}

Standard superconducting JJ circuits used for the qubit application and readout are presented in Fig. 1 and include: current-biased single JJ and dc SQUID, rf SQUID with one or more JJ, and the single Cooper pair box. These circuits consist of various combinations of the three basic elements: capacitive elements including Josephson junction capacitors, linear inductive elements of superconducting leads, and nonlinear inductances of Josephson tunnel junctions. All these circuits exhibit dynamical properties of a network of nonlinear oscillators. ${ }^{47}$

The possibility for macroscopic electrical circuits to exhibit quantum behavior is rather counterintuitive. However, it is, in fact, a consequence of the quantum origin of the electromagnetic field. The Kirchhoff equations used to describe these circuits represent a lumped element approximation of the Maxwell equations valid for the limit of small circuit size compared to the electromagnetic wavelength. Typical superconducting qubits operate at frequencies of several $\mathrm{GHz}$, which correspond to wavelengths in the centimeter range, while circuit elements are of a submillimeter size.

Quantum electrodynamics, translated to the language of lumped element circuits, establishes the non-commutation relations between the charges and the currents.

The quantum behavior of electrical circuits was appreciated and discussed back in the 1950s, in the context of electrical current fluctuation. ${ }^{48}$ However, the first observation of a real quantum effect, macroscopic quantum tunneling (MQT), was made only in 1981, when quantum switching of a tunnel junction from the Josephson regime to the dissipative regime was discovered. ${ }^{49}$

While having been convinced of the possibility of quantization in electrical circuits, one might be surprised that quantum effects are not commonly observed in conventional normal metal and semiconducting circuits: Indeed, in highfrequency applications, frequencies up to $\mathrm{THz}$ are available, which correspond to a distance between quantized oscillator levels of the order of $10 \mathrm{~K}$; this should be observable at temperature of tens of millikelvin. Furthermore, it is intuitively clear and follows from a rigorous analysis ${ }^{7}$ that the dissipation effects, which destroy the quantum coherence, are not efficient when the broadening of the energy levels due to dissipation is smaller than the distance between the levels.

This requirement can be easily fulfilled in resonators with high quality factors. In fact, the real difficulty for the observation of the quantum dynamics is related to the linear oscillator character of high quality LC-circuits: by virtue of the Ehrenfest theorem, ${ }^{50}$ the quantum dynamics and the classical dynamics of linear oscillators are not distinguishable.
For the quantum dynamics to be reliably observed, a nonlinear nondissipative circuit element is required; this is provided by the nonlinear inductance of the Josephson tunneling. For an illuminative discussion of this issue we refer to the paper by Martinis, Devoret, and Clarke. ${ }^{51}$

A basis for the quantum description of the qubit circuits is the Hamilton formalism. In the classical limit, the dynamical equations for the conjugate variables are equivalent to the standard Kirchhoff rules. The building blocks for constructing the circuit Hamiltonian are given by the kinetic energy associated with the charging energy of the capacitive elements, $K=C V^{2} / 2$, and the potential energy associated with the Josephson inductance, $U_{J}=-E_{J} \cos \phi$, and the inductance of the superconducting leads, $U_{L}=\Phi^{2} / 2 L^{52-54}$ All these quantities are to be expressed in terms of the superconducting phase difference $\phi$ for a given circuit element, whose connection to the voltage drop $V$ and magnetic flux $\Phi$ is established by the Josephson relations, $V=(\hbar / 2 e) \dot{\phi}$ and $\Phi$ $=(\hbar / 2 e)^{2} \phi$. In the Hamilton formalism, the kinetic energy is expressed through the momentum conjugate to the phase coordinate, conveniently defined as $n=\partial K(\dot{\phi}) /(\hbar \partial \dot{\phi})$. This momentum obeys the Poissonian bracket relation, $\left\{\phi_{j}, n_{k}\right\}$ $=(1 / \hbar) \delta_{j k}$, and has the physical meaning of the charge $q$ accumulated on the junction capacitor in units of the double electronic charge, $q=2 e n$, i.e., the number of the Cooper pairs stored on the capacitor. The circuit Hamiltonian is then constructed by summing up the energies of all the circuit elements,

$$
H=\sum\left(K\left(n_{j}\right)+U\left(\phi_{j}\right)\right) .
$$

If several circuit elements are connected in a closed loop, the flux quantization equation imposes a constraint on the phases of these elements: $\Sigma \phi_{i}+\phi_{e}=2 \pi n$, where $\phi_{e}$ $=(2 e / \hbar) \Phi_{e}$ is the phase associated with the applied magnetic flux.

The current-biased Josephson junction, Fig. 1a, is described with the Hamiltonian,

$$
H=E_{C} n^{2}-E_{J} \cos \phi-\frac{\hbar}{2 e} I_{e} \phi
$$

where $E_{C}=(2 e)^{2} / 2 C$ is the charging energy, $E_{J}=\left(\hbar^{2} / 2 e\right) I_{c}$ is the Josephson energy, $I_{c}$ is the critical Josephson current; $I_{e}$ indicates the applied current, which serves as a controlling parameter. The small amplitude electromagnetic oscillation in this circuit, plasma oscillation, has the frequency $\hbar \omega$ $=\sqrt{2 E_{C} E_{J}}\left(\right.$ at $\left.I_{e}=0\right)$.

The rf SQUID Hamiltonian, Fig. 1c, has the form,

$$
H=E_{C} n^{2}-E_{J} \cos \phi+E_{L} \frac{\left(\phi-\phi_{e}\right)^{2}}{2}
$$

here $E_{L}=\hbar^{2} /(2 e)_{L}^{2}$, and $\phi_{e}$ plays the role of controlling parameter.

The dc SQUID, shown in Fig. 1b, has two degrees of freedom, $\phi_{1,2}$, and its Hamiltonian can be written by combining Eqs. (1) and (2) in terms of the phases $\phi_{ \pm}=(1 / 2)$ $\times\left(\phi_{1} \pm \phi_{2}\right)$. In the symmetric case we have 


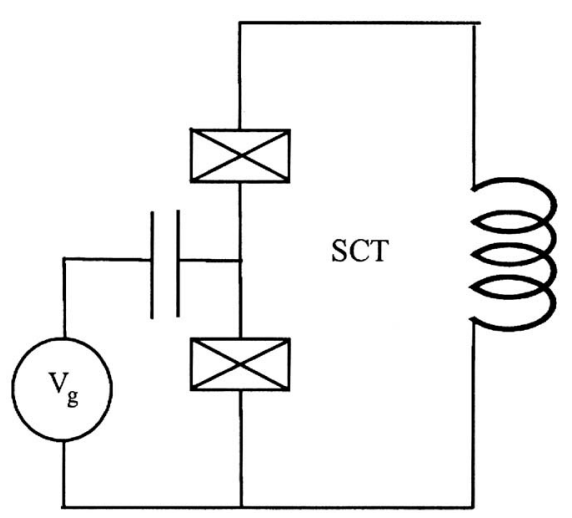

FIG. 2. Single Cooper pair transistor (SCT): SCB with loop-shape bulk electrode connected to the island via two JJs; charge fluctuation on the island produces current fluctuation in the loop.

$$
\begin{aligned}
H= & E_{C} n_{+}^{2}+E_{C} n_{-}^{2}-2 E_{J} \cos \phi_{+} \cos \phi_{-} \\
& +E_{L} \frac{\left(2 \phi_{+}-\phi_{e}\right)^{2}}{2}+\frac{\hbar}{2 e} I_{e} \phi_{-} .
\end{aligned}
$$

This circuit is often used for qubit measurements.

The single Cooper pair box, shown in Fig. 1d, consists of a small superconducting island coupled to a massive electrode via a small resistive JJ, and also capacitively coupled to an electrostatic gate; the gate potential is controlled by a voltage source $V_{g}$. The classical Hamiltonian for this circuit has the form

$$
H=E_{C}\left(n-n_{g}\right)^{2}-E_{J} \cos \phi,
$$

where $n_{g}=-C_{g} V_{g} / 2 e$ plays the role of external controlling parameter, and $C_{g}$ is the gate capacitance. The name of the circuit stems from the analogous normal metallic circuit, the single electron box (SEB). ${ }^{55,56}$ If the tunnel junction resistance exceeds the quantum resistance $R_{q} \approx 26 \mathrm{~K} \Omega$, and the temperature is small compared to the charging energy of the island, the system is in the Coulomb blockade regime: ${ }^{57,58}$ the electrons can be transferred to the island one by one, the number of electrons on the island being controlled by the gate voltage. In the superconducting state, the number of electrons on the island changes pairwise. ${ }^{18,19,59}$ To achieve such a regime, one has to take into account the parity effect, ${ }^{59}$ a difference between the energies of even and odd numbers of electrons on the island. While an electron pair belongs to the superconducting condensate and has the additional charging energy $E_{C}$, a single electron forms an excitation, and thus its energy consists of the charging energy $E_{C} / 2$ plus the excitation energy $\Delta$. To provide the SCB regime and prevent the appearance of individual electrons on the island, the condition $\Delta \gg E C_{/} 2$ must be fulfilled.

The single Cooper pair transistor (SCT), a useful modification of the SCB, is presented in Fig. 2; here the island is connected to the electrode via two Josephson junctions. The advantage of this circuit is the possibility of tuning the effective Josephson energy of the SCB by applying magnetic flux to the circuit loop, similar to the dc SQUID. Also, the charge on the island is related to the magnitude of the induced persistent current circulating in the loop, which can be used for the measurement of the charge state of the island.
The classical Hamiltonian for this circuit is a combination of Eqs. (2) and (4), and has the form

$$
\begin{aligned}
H_{S C T}= & E_{C}\left(n_{-}-n_{g}\right)^{2}+E_{C} n_{+}^{2}-2 E_{J} \cos \phi_{+} \cos \phi_{-} \\
& +E_{L} \frac{\left(2 \phi_{+}-\phi_{e}\right)^{2}}{2} .
\end{aligned}
$$

Technically, the quantization of electrical circuits is introduced by generalizing the Poisson bracket relation $\left[\phi_{j}, n_{k}\right]=i \delta_{j k}$. This quantization rule is satisfied, as in the Schrödinger quantum mechanics, by substituting the momentum $n$ with the operator $n=-i \partial / \partial \phi$ in Eqs. (1)-(4). ${ }^{52-54}$

The quantum dynamics of an isolated $\mathrm{JJ}$ is described with the Mathieu-Bloch picture for a particle moving in a periodic potential, similar to the electronic solid state theory. ${ }^{17}$ Two limiting regimes are usually distinguished: the phase regime, $E_{J} \gg E_{C}$, is analogous to the tight-binding approximation, and the charge regime, $E_{J} \ll E_{C}$, is analogous to the nearly free particle approximation. In the phase regime, the quantum particle representing the $\mathrm{JJ}$ is basically confined to a single potential well; the well contains many energy levels since $\hbar \omega \ll E_{J}$. This regime is the closest to the junction classical dynamics. For the lowest energy levels the parabolic approximation for the junction potential is appropriate, giving the level spacing $\Delta E_{n} \approx \hbar \omega$. However, nonequidistance of the energy spectrum is essential, allowing the selection of two energy levels for qubit operation. Phase qubits and flux qubits usually operate in this phase regime.

In the charge regime, the junction eigenstate wave functions are close to the plane waves, $\exp [i(q / 2 e) \phi]$, where $q$ has the meaning of the charge on the junction capacitor (quasi-charge). In the specific case of the SCB, this quantity corresponds to the charge on the island, which must be equal to an integer number of electron pairs. This charge quantization requirement is fulfilled by imposing a periodic boundary condition on the junction wave function,

$$
\psi(\phi)=\psi(\phi+2 \pi) .
$$

This implies that an arbitrary state of the SCB is a superposition of charge states with integer numbers of Cooper pairs,

$$
\psi(\phi)=\sum_{n} a_{n} \exp (\operatorname{in} \phi) .
$$

For half-integer values of the gate charge, $n_{g}=n+1 / 2$, the two neighboring charge states are almost degenerate and separated by a small energy interval $E_{J} \ll E_{C}$. Charge qubits usually operate in this charge regime, the two tight levels $n$ $=0,1$ in the vicinity of $n_{g}-=1 / 2$, being usually selected as the qubit states.

\section{BASIC QUBITS}

The quantum superconducting circuits considered above contain a large number of energy levels, while for qubit operation only two levels are required. Moreover, these two qubit levels must be well decoupled from the other levels in the sense that transitions between qubit levels and the environment must be much less probable than the transitions between the qubit levels themselves. Typically that means that 


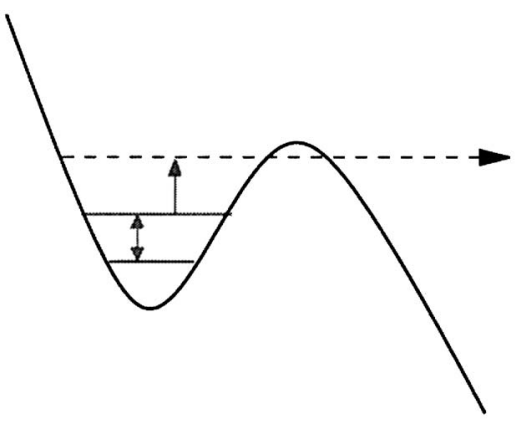

FIG. 3. Quantized energy levels in the potential of a current-biased JJ; the two lower levels form the JJ qubit, the dashed line indicates a leaky level with higher energy.

the qubit should involve a low-lying pair of levels, well separated from the spectrum of higher levels, and not being close to resonance with any other transitions.

\section{Single Josephson junction qubit}

The simplest qubit realization is a current-biased JJ with large Josephson energy compared to the charging energy. In the classical regime, the particle representing the phase either rests at the bottom of one of the wells of the tilted cosine potential ("washboard" potential), or oscillates within the well.

Due to the periodic motion, the average voltage across the junction is zero: $\phi=0$. Strongly excited states, where the particle may escape from the well, correspond to the dissipative regime with nonzero average voltage across the junction, $\dot{\phi} \neq 0$.

In the quantum regime described by the Hamiltonian (1), particle confinement, rigorously speaking, is impossible because of MQT through the potential barrier; see Fig. 3. However, the probability of MQT is small and the tunneling may be neglected if the particle energy is close to the bottom of the local potential well, i.e., when $E \ll E_{J}$. To find the conditions for such a regime, it is convenient to approximate the potential with a parabolic function, $U(\phi)$ $\approx(1 / 2) E_{J} \cos \phi_{0}\left(\phi-\phi_{0}\right)^{2}$, where $\phi_{0}$ corresponds to the potential minimum, $E_{J} \sin \phi_{0}=(\hbar / 2 e) I_{e}$. Then the lowest energy levels, $E_{k}=\hbar \omega_{p}(k+1 / 2)$ are determined by the plasma frequency, $\omega_{p}=2^{1 / 4} \omega_{J}\left(1-I_{e} / I_{c}\right)^{1 / 4}$. It then follows that the levels are close to the bottom of the potential if $E_{C} \ll E_{J}$, i.e., when the $\mathrm{JJ}$ is in the phase regime, and moreover, if the bias current is not too close to the critical value, $I_{e}<I_{c}$.

It is essential for qubit operation that the spectrum in the well is not equidistant. Then the two lowest energy levels, $k=0,1$, can be employed for the qubit operation. Truncating the full Hilbert space of the junction to the subspace spanned by these two states, $|0\rangle$ and $|1\rangle$, we may write the qubit Hamiltonian in the form

$$
H_{q}=-\frac{1}{2} \varepsilon \sigma_{z},
$$

where $\varepsilon=E_{1}-E_{2}$.

The interlevel distance is controlled by the bias current. When bias current approaches the critical current, level broadening due to MQT starts to play a role, $E_{k} \rightarrow E_{k}$ $+i \Gamma_{k} / 2$. The MQT rate for the lowest level is given by ${ }^{60}$

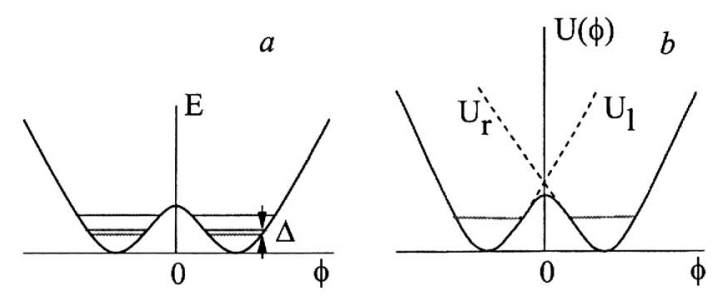

FIG. 4. Double-well potential of the rf SQUID with degenerate quantum levels in the wells. Macroscopic quantum tunneling through the potential barrier introduces a level splitting $\Delta$, and the lowest level pair forms a qubit (a); truncation of the junction Hamiltonian, dashed lines indicate potentials of the left and right wells with ground energy levels $(b)$.

$$
\Gamma_{M Q T}=\frac{52 \omega_{p}}{2 \pi} \sqrt{\frac{U_{\max }}{\hbar \omega_{p}}} \exp \left(-\frac{7.2 U_{\max }}{\hbar \omega_{p}}\right),
$$

where $U_{\max }=2 \sqrt{2}\left(\Phi_{0} / 2 \pi\right)\left(1-I_{e} / I_{c}\right)^{3 / 2}$ is the height of the potential barrier at given bias current.

\section{Flux qubit}

An elementary flux qubit can be constructed with an rf SQUID operating in the phase regime, $E_{J} \gg E_{C}$. Let us consider the Hamiltonian (2) at $\phi_{e}=\pi$, i.e., at half-integer bias magnetic flux. The potential $U(\phi)$ shown in Fig. 4 has two identical wells with equal energy levels when MQT between the wells is neglected (phase regime, $\omega_{J} \ll E_{J}$ ). These levels are connected with current fluctuations within each well around averaged values corresponding to clockwise and counterclockwise persistent currents circulating in the loop (the flux states). Let us consider the lowest, doubly degenerate, energy level. When the tunneling is switched on, the levels split, and a tight two-level system is formed with the level spacing determined by the MQT rate, which is much smaller than the level spacing in the well.

In the case that the tunneling barrier is much smaller than the Josephson energy, the potential in Eq. (2) can be approximated as

$$
\begin{aligned}
U(\phi) & =E_{J}(1-\cos \phi)+E_{L} \frac{\left(\phi-\phi_{e}\right)^{2}}{2} \\
& \approx E_{L}\left(-\in \frac{\widetilde{\phi}^{2}}{2}-f \widetilde{\phi}+\frac{1+\in}{24} \widetilde{\phi}^{4}\right),
\end{aligned}
$$

where $\widetilde{\phi}=\phi-\pi, f=\phi_{e}-\pi$, and where the parameter $\epsilon$ $=\left(E_{J} / E_{L}\right)-1 \ll 1$ determines the height of the tunnel barrier.

The qubit Hamiltonian is derived by projecting the full Hilbert space of the Hamiltonian (2) on the subspace spanned by these two levels. The starting point of the truncation procedure is to approximate the double-well potential with $U_{l}$ and $U_{r}$, as shown in Fig. 4, to confine the particle to the left or to the right well, respectively. The corresponding ground-state wave functions $|l\rangle$ and $|r\rangle$ satisfy the stationary Schrödinger equation

$$
H_{l}|l\rangle=E_{l}|l\rangle, \quad H_{r}|r\rangle=E_{r}|r\rangle .
$$

The averaged induced flux for these states, $\phi_{l}$ and $\phi_{r}$, has opposite sign, manifesting opposite directions of the circulating persistent currents. Let us allow the bias flux to deviate slightly from the half-integer value, $\phi_{e}=\pi+f$, so that the ground-state energies are not equal but still close to each 


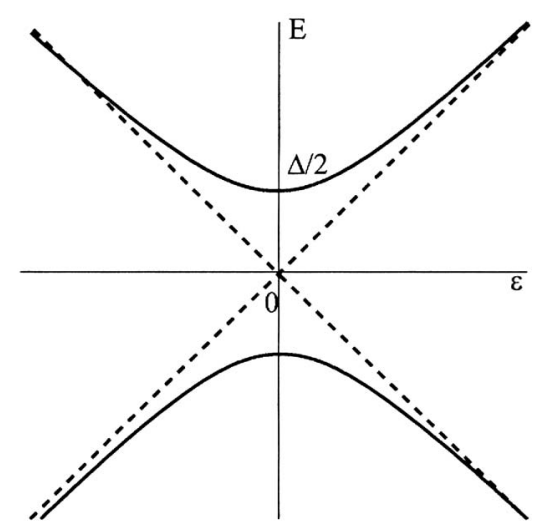

FIG. 5. Energy spectrum of the flux qubit versus bias flux (solid lines): it results from hybridization of the flux states (dashed lines).

other, $E_{l} \approx E_{r}$. The tunneling will hybridize the levels, and we can approximate the true eigenfunction $|E\rangle$,

$$
H|E\rangle=E|E\rangle,
$$

with a superposition, $|E\rangle=a|l\rangle+b|r\rangle$. The qubit Hamiltonian is given by the matrix elements of the full Hamiltonian, Eq. (12), with respect to the states $|l\rangle$ and $|r\rangle$ :

$$
\begin{aligned}
& H_{l l}=E_{l}+\left\langle l\left|U-U_{l}\right| l\right\rangle, \quad H_{r r}=E_{r}+\left\langle r\left|U-U_{r}\right| r\right\rangle, \\
& H_{r l}=E_{l}\langle r \mid l\rangle+\left\langle r\left|U-U_{l}\right| l\right\rangle .
\end{aligned}
$$

In the diagonal matrix elements, the second terms are small because the wave functions are exponentially small in the region where the deviation of the approximated potential from the true potential is appreciable. The off diagonal matrix element is exponentially small because of small overlap of the ground-state wave functions in the left and right wells, and also here the main contribution comes from the first term. Since the wave functions can be chosen real, the truncated Hamiltonian is symmetric, $H_{l r}=H_{r l}$. Then introducing $\varepsilon=E_{r}-E_{l} \propto f$ and $\Delta / 2=H_{r l}$, we arrive at the Hamiltonian of the flux qubit,

$$
H=-\frac{1}{2}\left(\varepsilon \sigma_{z}+\Delta \sigma_{x}\right)
$$

The energy spectrum of the flux qubit is given by the equation,

$$
E_{1,2}=\mp \frac{1}{2} \sqrt{\varepsilon^{2}+\Delta^{2}}
$$

as shown in Fig. 5. The energy levels are controlled by the bias magnetic flux. The dashed lines refer to the persistent current states in the absence of macroscopic tunneling. These states, $|l\rangle$ and $|r\rangle$, form the basis of the qubit Hamiltonian in Eq. (13), and correspond to certain values of the induced flux, $\phi_{l}$ and $\phi_{r}$. Far from the degeneracy point $(\varepsilon=0$, corresponding to a half-integer bias flux, $\phi_{e}=\pi$ ) the qubit eigenstates are almost pure flux states. At the flux degeneracy point, the expectation value of the induced flux is equal zero, and the qubit eigenstates are given by equal-weight superpositions of the flux states, $\left|E_{1}\right\rangle,\left|E_{2}\right\rangle=|l\rangle \mp|r\rangle$ (cat states). The level spacing at this point is determined by the small ampli-
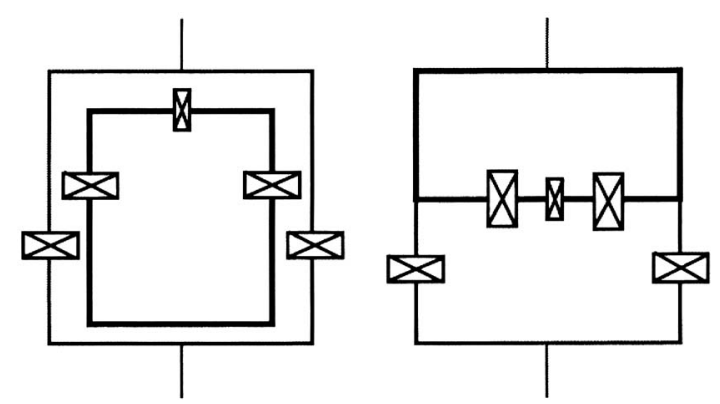

FIG. 6. Persistent current flux qubit with 3 junctions (bold line) connected inductively (left), and galvanically (right) to a measurement dc SQUID.

tude of the tunneling through the macroscopic potential barrier.

The possibility of achieving quantum coherence of macroscopic current states in an rf SQUID with a small capacitance Josephson junction was first pointed out in 1984 by Leggett. ${ }^{4}$ However, successful experimental observation of the effect was achieved only in 2000, by Friedman et al. ${ }^{13}$

\section{Flux qubit with 3 junctions}

The main drawback of the flux qubit with a single Josephson junction ( $r f$ SQUID) described above concerns the large inductance of the qubit loop, the energy of which must be comparable to the Josephson energy to form the required double-well potential profile. This implies a large size of the qubit loop, which makes the qubit vulnerable to dephasing by magnetic fluctuations of the environment. One way to overcome this difficulty was pointed out by Mooij et al.: ${ }^{11}$ replacing the large loop inductance by the Josephson inductance of an additional tunnel junction, as shown in Fig. 6.

The design employs three tunnel junctions connected in series in a superconducting loop. The inductive energy of the loop is chosen to be much smaller than the Josephson energy of the junctions. Two junctions are identical, while the third junction has a smaller area, and therefore a smaller Josephson and a larger charging energy. The Hamiltonian has the form

$$
\begin{aligned}
H= & E_{C}\left[n_{1}^{2}+n_{2}^{2}+\frac{n_{3}^{2}}{1 / 2+\varepsilon}\right]-E_{J}\left[\cos \phi_{1}+\cos \phi_{2}\right. \\
& \left.+(1 / 2+\varepsilon) \cos \phi_{3}\right] .
\end{aligned}
$$

To explain the idea, let us consider the potential energy. The three phases are not independent and satisfy the relation $\phi_{1}$ $+\phi_{2}+\phi_{3}=\phi_{e}$. Let us suppose that the qubit is biased at halfinteger flux quantum, $\phi_{e}=\pi$. Then introducing the new variables $\phi_{ \pm}=\left(\phi_{1} \pm \phi_{2}\right) / 2$, we have

$$
U\left(\phi_{+}, \phi_{-}\right)=-E_{J}\left[2 \cos \phi_{-} \cos \phi_{+}-(1 / 2+\varepsilon) \cos 2 \phi_{+}\right] .
$$

The two-dimensional periodic potential landscape of this circuit contains the double-well structures near the points $\left(\phi_{+}, \phi_{-}\right)=(0,0) \bmod 2 \pi$. An approximate form of the potential energy structures is given by 


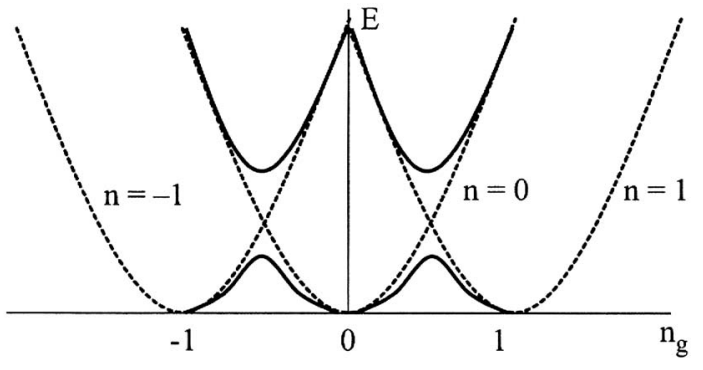

FIG. 7. SCB energy spectrum (bold) versus gate potential: it results from hybridization of the charge states (dashed) due to Josephson tunneling; level anticrossings occur at $n_{g}=n+1 / 2$.

$$
U\left(\phi_{+}, 0\right) \approx E_{J}\left(-2 \varepsilon \phi_{+}^{2}+\frac{\phi_{+}^{4}}{4}\right)
$$

Each well in this structure corresponds to clockwise and counterclockwise currents circulating in the loop. The amplitude of the structure is given by the parameter $\varepsilon E_{J}$, and for $\varepsilon \ll 1$ the tunneling between these wells dominates. Thus this qubit is qualitatively similar to the single-junction qubit described above, but the quantitative parameters are different and can be significantly optimized.

\section{Charge qubit-SCB}

An elementary charge qubit can be made with the SCB operating in the charge regime, $E_{C} \gg E_{J}$. Neglecting the Josephson coupling implies complete isolation of the island of the SCB, with a specific number of Cooper pairs trapped on the island. Correspondingly, the eigenfunctions,

$$
E_{C}\left(n-n_{g}\right)^{2}|n\rangle=E_{n}|n\rangle,
$$

correspond to the charge states $n=0,1,2 \ldots$, with the energy spectrum $E_{n}=E_{C}\left(n-n_{g}\right)^{2}$, as shown in Fig. 7. The groundstate energy oscillates with the gate voltage, and the number of Cooper pairs in the ground state increases. There are, however, specific values of the gate voltage, e.g., $n_{g}=1 / 2$, where the charge states $|0\rangle$ and $|1\rangle$ become degenerate. Switching on a small Josephson coupling will then lift the degeneracy, forming a tight two-level system.

The qubit Hamiltonian is derived by projecting the full Hamiltonian (4) on the two charge states, $|0\rangle,|1\rangle$, leading to:

$$
H_{S C B}=-\frac{1}{2}\left(\varepsilon \sigma_{z}+\Delta \sigma_{x}\right),
$$

where $\varepsilon=E_{C}\left(1-2 n_{g}\right)$, and $\Delta=E_{J}$. The qubit level energies are then given by the equation

$$
E_{1,2}=\mp \frac{1}{2} \sqrt{E_{C}^{2}\left(1-2 n_{g}\right)^{2}+E_{J}^{2}},
$$

the interlevel distance being controlled by the gate voltage. At the degeneracy point, $n_{g}=1 / 2$, the diagonal part of the qubit Hamiltonian vanishes, the levels being separated by the Josephson energy, $E_{J}$, and the qubit eigenstates corresponding to the cat states, $\left|E_{1}\right\rangle,\left|E_{2}\right\rangle=|0\rangle \mp|1\rangle$. For these states, the average charge on the island is zero, while it changes to $\mp 2 e$ far from the degeneracy point, where the qubit eigenstates approach pure charge states.
The SCB was first experimentally realized by Lafarge $e t$ al. ${ }^{19}$ who observed a Coulomb staircase with steps of $2 e$ and a superposition of the charge states; see also Ref. 20.

Realization of the charge qubit by manipulation of the SCB and observation of Rabi oscillations was first achieved by Nakamura et al., ${ }^{6,61,62}$ and further investigated theoretically by Choi et al. ${ }^{63}$

\section{Charge-phase qubit SCT}

In the SCB, the charge fluctuation on the island generates fluctuating current between the island and bulk electrode. In the two-junction setup, Fig. 2, an interesting question concerns how the current is distributed between the two junctions.

Answering to this question is apparently equivalent to evaluating the persistent current circulating in the SCT loop. For small but nonzero inductance of the loop, the amplitude of the induced phase is small, $\widetilde{\phi}=2 \phi_{+}-\phi_{e} \ll 1$, and the cosine term in Eq. (5) containing $\phi_{+}$can be expanded, yielding the equation

$$
H_{S C T}=H_{S C B}\left(\phi_{-}\right)+H_{\mathrm{osc}}(\widetilde{\phi})+H_{\mathrm{int}} .
$$

$H_{S C B}\left(\phi_{-}\right)$is the SCB Hamiltonian (4) with the fluxdependent Josephson energy, $E_{J}\left(\phi_{e}\right)=2 E_{J} \cos \left(\phi_{e} / 2\right)$. $H_{\text {osc }}(\widetilde{\phi})$ describes the linear oscillator associated with the variable, $H_{\mathrm{osc}}(\widetilde{\phi})=4 E_{C} \widetilde{n}^{2}+E_{L} \widetilde{\phi}^{2} / 2$, and the interaction term reads: $H_{\mathrm{int}}=E_{J} \sin \left(\phi_{e} / 2\right) \cos \left(\phi_{-}\right) \tilde{\phi}$. Thus the circuit consists of the nonlinear oscillator of the SCB linearly coupled to the linear oscillator of the SQUID loop. This coupling gives the possibility of measuring the charge state of the SCB by measuring the persistent currents and the induced flux.

Truncating Eq. (21), we finally arrive at the Hamiltonian which is formally equivalent to the spin-oscillator Hamiltonian:

$$
H_{S C T}=-\frac{1}{2}\left(\varepsilon \sigma_{z}+\Delta\left(\phi_{e}\right) \sigma_{x}\right)+\lambda \widetilde{\phi} \sigma_{x}+H_{\mathrm{osc}} .
$$

In this equation, $\Delta\left(\phi_{e}\right)=2 E_{J} \cos \left(\phi_{e} / 2\right)$, and $\lambda$ $=E_{J} \sin \left(\phi_{e} / 2\right)$.

\section{Potential superconducting qubits}

The superconducting qubits that have been discussed in previous Sections exploit the fundamental quantum uncertainty between electric charge and magnetic flux. There are, however, other possibilities. One of them is to delocalize quantum information in a JJ network by choosing global quantum states of the network as a computational basis. Recently, some rather complicated JJ networks have been discussed, which have the unusual property of a degenerate ground state, which might be employed for efficient qubit protection against decoherence. ${ }^{64,65}$

An alternative possibility is to replace the macroscopic tunnel Josephson junction with a single-mode quantum point contact (QPC), and to take advantage of quantum fluctuation of microscopic bound Andreev states controlling the Josephson current. ${ }^{66,67}$ 


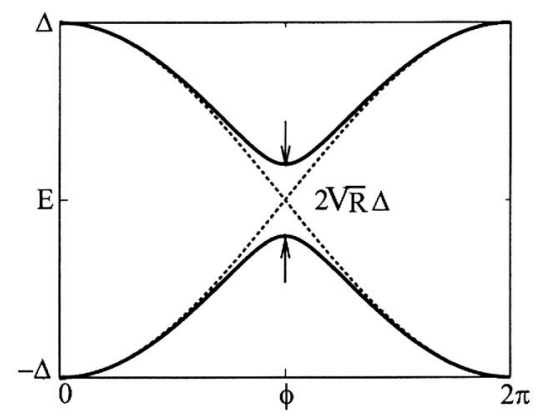

FIG. 8. Energy spectrum of microscopic bound Andreev levels; the level splitting is determined by the contact reflectivity.

\section{Andreev level qubit}

To explain the physics of this type of device, let us consider an rf SQUID, Fig. 1c, with a point contact junction that has such a small cross section that the quantization of electronic modes in the direction perpendicular to the current flow becomes pronounced. In such a QPC junction the Josephson current is carried by a number of independent conducting electronic modes, each of which can be considered an elementary microscopic Josephson junction characterized by its own transparency.

The number of modes is roughly proportional to the ratio of the junction cross section and the area of the atomic cell (determined by the Fermi wavelength) of the junction material. In an atomic-size QPC with only a few conducting modes, the Josephson current can be appreciable if the conducting modes are transparent open modes. If the junction reflectivity is zero $(R=0)$ then current is a well-defined quantity. This will correspond to a persistent current with certain direction circulating in the qubit loop. On the other hand, for a finite reflectivity, $R \neq 0$, the electronic backscattering will induce hybridization of the persistent current states, giving rise to strong quantum fluctuation of the current.

Such a quantum regime is distinctly different from the macroscopic quantum coherence regime of the flux qubit, where the quantum hybridization of the persistent current states is provided by the charge fluctuation on the junction capacitor. In QPC the leading role belongs to the microscopic mechanism of electron backscattering, while charging effects do not play any essential role. On the other hand, in the large-area junctions of macroscopic qubits, the microscopic quantum fluctuation of the Josephson current is negligibly small, since the current here is carried by a large number $\left(>10^{4}\right)$ of statistically independent conducting modes.

In a QPC, the Josephson effect is associated with microscopic Andreev levels, localized in the junction area, which transport Cooper pairs from one junction electrode to the other. ${ }^{68,69}$ As shown in Fig. 8, the Andreev levels lie within the superconducting gap and have the phase-dependent energy spectrum

$$
E_{a}=\Delta \sqrt{\cos ^{2}(\phi / 2)+R \sin ^{2}(\phi / 2)}
$$

(here $\Delta$ is the superconducting order parameter in the junction electrodes). For very small reflectivity, $R \ll 1$, and phase close to $\pi$ (half-integer flux bias) the Andreev two-level system is well isolated from the continuum states. The expecta- tion value for the Josephson current carried by the level is determined by the Andreev level spectrum,

$$
I_{a}=\frac{2 e}{\hbar} \frac{d E_{a}}{d \phi_{e}}
$$

and it has different sign for the upper and lower level. Since the state of the Andreev two-level system is determined by the phase difference and is related to the Josephson current, the state can be manipulated by driving magnetic flux through the SQUID loop, and read out by measuring the circulating persistent current. $^{70,71}$

This microscopic physics underlies a proposal for an Andreev level qubit. ${ }^{66,67}$ The qubit is similar to the macroscopic flux qubits with respect to how it is manipulated and measured, but the great difference is that the quantum information is stored in the microscopic quantum states. This difference is reflected in the more complex form of the qubit Hamiltonian, which consists of the two-level Hamiltonian of the Andreev levels strongly coupled to the quantum oscillator describing phase fluctuations,

$$
H=\Delta \mathrm{e}^{-i \sigma_{x} \sqrt{R} \phi / 2}\left(\cos \frac{\phi}{2} \sigma_{z}+\sqrt{R} \sin \frac{\phi}{2} \sigma_{y}\right)+H_{\mathrm{osc}}[\phi],
$$

$H_{\mathrm{osc}}[\phi]=E_{C} n+\left(E_{L} / 2\right)\left(\phi-\phi_{e}\right)^{2}$. Comparing this equation with, e.g., the SCT Hamiltonian (21), we find that the truncated Hamiltonian of the SCB is replaced here by the Andreev level Hamiltonian.

Bound Andreev levels in a QPC offer yet another interesting possibility for the qubit. ${ }^{72}$ In the presence of a Zeeman magnetic field, Andreev levels may undergo spin polarization, which gives the possibility of exciting the spin dynamics using NMR-type technique. Similar to the Andreev level qubit, transitions between the spin polarized Andreev states induce oscillation of the Josephson current and thus can be detected.

\section{QUBIT OPERATION AND DECOHERENCE}

\section{Qubit operation}

Quantum computation basically means allowing the $N$-qubit state to develop in a fully coherent fashion through unitary transformations acting on all $\mathrm{N}$ qubits. ${ }^{1}$ The difference from the conventional many-body problem is that this evolution must be controlled according to the prescriptions of a quantum algorithm. An arbitrary quantum algorithm can be implemented through a set of elementary operationsuniversal gates-with single qubits and coupled qubit pairs. ${ }^{2}$ Therefore a universal quantum computer is represented by the Hamiltonian of a pseudospin-1/2 array with controllable spin-spin interactions subject to a variable local "magnetic" field:

$$
\begin{aligned}
H= & -\frac{1}{2} \sum_{i}\left[\varepsilon_{i}(t) \sigma_{i z}+\Delta_{i}^{\prime}(t) \sigma_{i x}+\Delta_{i}^{\prime \prime}(t) \sigma_{i y}\right] \\
& +\frac{1}{2} \sum_{i j} \lambda_{i j}^{\nu}(t) ; \sigma_{i \nu} \sigma_{j \nu} .
\end{aligned}
$$

A set of universal single qubit gates include qubit rotations around 3 axes, $x, y, z$, allowing the pseudospin to reach any point on the Bloch sphere; see Fig. 9. For superconduct- 

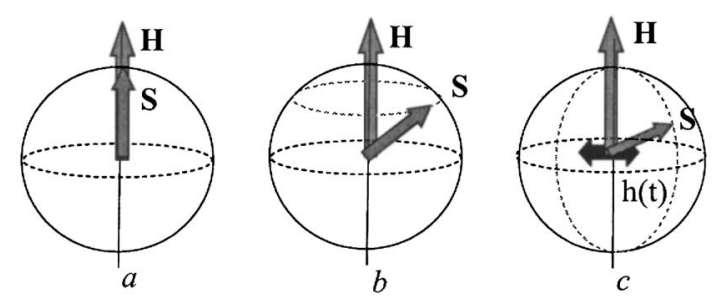

FIG. 9. The Bloch sphere: the Bloch vector $\mathbf{S}$ represents the states of the two-level system; the vector $\mathbf{H}$ represents the two-level Hamiltonian; the Bloch vector of the energy eigenstate is parallel (antiparallel) to the vector $\mathbf{H}(a)$; free evolution of the Bloch vector (precession) (b); rotation of the Bloch vector under a time dependent perturbation-Rabi oscillation $(c)$.

ing qubits, such rotations can be achieved by pulsing the controlling physical parameters: the applied current for the JJ phase qubits, applied magnetic flux for the flux qubits, and electrostatic gate potential for the charge qubits. Defining the $z$ axis as pointing along the energy eigenstate direction, we find that the $z$ rotation is simply realized by free qubit evolution. Rotations around perpendicular axes are usually performed by applying rf pulses with small amplitudes and resonance frequency with respect to the free qubit rotation, inducing Rabi oscillation between the qubit eigenstates (NMR-type operation). ${ }^{73}$

\section{Decoherence of qubit systems}

Ideally, a quantum computer is supposed to evolve maintaining a pure entangled state of $N$ qubits under a unitary transformation. However, in practice, the quantum coherence is destroyed by qubit environment. For macroscopic superconducting qubits, the environment basically consists of various dissipative elements in external circuits which provide bias, control, and measurement of the qubit. The "offchip" parts of these circuits are usually kept at room temperature and produce significant noise. Examples are the fluctuations in the current source producing magnetic field to bias flux qubits and, similarly, fluctuations of the voltage source to bias gate of the charge qubits. Electromagnetic radiation from the qubit during operation is another dissipative mechanism. There are also intrinsic microscopic mechanisms of decoherence, such as fluctuating trapped charges in the substrate of the charge qubits, and fluctuating trapped magnetic flux in the flux qubits, believed to produce dangerous $1 / \mathrm{f}$ noise. Other intrinsic mechanisms concern the losses in the dielectric layer of the tunnel junction. ${ }^{35,74-76}$

Various kinds of environment are commonly modeled with an infinite set of linear oscillators in thermal equilibrium (thermal bath), linearly coupled to the qubit (CaldeiraLeggett model $\left.^{7,10}\right)$. The extended qubit-plus-environment Hamiltonian has the following form in the qubit energy eigenbasis: ${ }^{60}$

$$
\begin{aligned}
H= & -\frac{1}{2} E \sigma_{z}+\sum_{i}\left(\lambda_{i z} \sigma_{z}+\lambda_{i \perp} \sigma_{\perp}\right) X_{i} \\
& +\sum_{i}\left(\frac{P_{i}^{2}}{2 m}+\frac{m \omega_{i}^{2} X_{i}^{2}}{2}\right)
\end{aligned}
$$

The physical effects of the two coupling terms in Eq. (27) are quite different. The "transverse" coupling term proportional to $\lambda_{\perp}$ induces interlevel transitions and eventually leads to relaxation. The "longitudinal" coupling term proportional to $\lambda_{z}$ commutes with the qubit Hamiltonian and thus does not induce interlevel transitions. However, it randomly changes the level spacing, which eventually leads to the loss of phase coherence: dephasing.

The effect of both processes, relaxation and dephasing, are referred to as decoherence. The time evolution of a qubit coupled to a bath is given, in the simplest approximation, by the Bloch-Redfield equations: ${ }^{73,77}$

$$
\partial_{t} \rho_{z}=-\frac{1}{T_{1}}\left(\rho_{z}-\rho_{z}^{(0)}\right), \quad \partial_{t} \rho_{12}=\frac{i}{\hbar} E \rho_{12}-\frac{1}{T^{2}} \rho_{12} .
$$

The first equation describes relaxation of the level population to the equilibrium form, $\rho_{z}^{(0)}=-(1 / 2) \tanh (E / k T), T_{1}$ being the relaxation time. The second equation describes disappearance of the off-diagonal matrix element during a characteristic time $T_{2}$-dephasing. The relaxation time is determined by the spectral density of the environmental fluctuations at the qubit frequency, $1 / T_{1}=\left(\lambda_{\perp}^{2} / 2\right) S_{\phi}(\omega=E)$. The particular form of the spectral density depends on the properties of the environment, which are frequently expressed via the impedance (response function) of the environment. The most common environment consists of a pure resistance; in this case $S_{\phi}(\omega) \propto \omega$ at low frequencies. The dephasing time consists of two parts, $1 / T_{2}=1 / 2 T_{1}+1 / T_{\phi}$. The first part is generated by the relaxation process, while the second part results from the pure dephasing due to the longitudinal coupling to the environment. This pure dephasing part is proportional to the spectral density of the fluctuation at zero frequency: $1 / T_{\phi}=\left(\lambda_{z}^{2} / 2\right) S_{\phi}(\omega=0)$. There is already a vast recent literature on decoherence and noise in superconducting circuits, qubits, and detectors, and how to engineer the qubits and environment to minimize decoherence and relaxation. $44,67,76-100$

\section{QUBIT READOUT}

In this Section we present a number of proposed and realized schemes for measuring quantum states of various superconducting qubits. The ultimate objective of a qubit readout device is to distinguish the eigenstates of a qubit in a single measurement "without destroying the qubit," a socalled "single-shot" quantum non-demolition (QND) projective measurement. This objective is essential for several reasons: state preparation for computation, readout for error correction during the calculation, and readout of results at the end of the calculation. Strictly speaking, the QND property is only needed if the qubit must be left in an eigenstate after the readout. In a broader sense, readout of a specific qubit must of course not destroy any other qubits in the system.

It must be carefully noted that one cannot "read out the state of a qubit" in a single measurement-this is prohibited by quantum mechanics. It takes repeated measurements on a large number of replicas of the quantum state to characterize the state of the qubit- "quantum tomography." "101

The measurement connects the qubit with the open system of the detector, which collapses the combined system of qubit and measurement device to one of its common eigenstates. If the coupling between the qubit and the detector is 
weak, the eigenstates are approximately those of the qubit. In general, however, one must consider the eigenstates of the total qubit-detector system and manipulate gate voltages and fluxes so that the readout measurement is performed in a convenient energy eigenbasis (see, e.g., Refs. 44 and 102).

Even under ideal conditions, a single-shot measurement can only determine the population of an eigenstate if the system is prepared in an eigenstate: then the answer will always be either " 0 " or " 1 ." If an ideal single-shot measurement is used to read out a qubit superposition state, e.g., during Rabi oscillation, then again the answer can only be "0" or "1." To determine the qubit population (i.e., the $\left|a_{1}\right|^{2}$ and $\left|a_{2}\right|^{2}$ probabilities) requires repetition of the measurement to obtain the expectation value. During the intermediate stages of quantum computation one must therefore not perform a measurement on a qubit unless one knows, because of the design and timing of the algorithm, that this qubit is in an energy eigenstate. Then the value is predetermined and the qubit left in the eigenstate (Stern-Gerlach-style).

On the other hand, to extract the desired final result it may be necessary to create an ensemble of calculations to be able to perform a complete measurement to determine the expectation values of variables of interest, performing quantum state tomography. ${ }^{101}$

\section{Direct qubit measurement}

Direct destructive measurement of the qubit can be illustrated with the example of a single JJ phase qubit. After the manipulation has been performed (e.g., Rabi oscillation), the qubit is left in a superposition of the upper and lower energy states. To determine the probability of the upper state, one slowly increases the bias current until it reaches such a value that the upper energy level equals (or gets close to) the top of the potential barrier (see Fig. 3). Then the junction, being at the upper energy level, will switch from the Josephson branch to the dissipative branch, and this can be detected by measuring the finite average voltage appearing across the junction (voltage state). If the qubit is in the lower energy state the qubit will remain on the Josephson branch and a finite voltage will not be detected (zero-voltage state). An alternative method to activate switching ${ }^{14}$ is to apply an $\mathrm{rf}$ signal with resonant frequency (instead of tilting the junction potential) in order to excite the upper energy level and to induce the switching event; see Fig. 3 (also illustrating a standard readout method in atomic physics).

It is obvious that, in this example, the qubit upper energy state is always destroyed by the measurement. Single-shot measurement is possible provided the MQT rate for the lower energy level is sufficiently low to prevent the junction switching during the measurement time. It is also essential to keep a sufficiently small rate of interlevel transitions induced by fluctuations of the bias current and by the current ramping.

A similar kind of direct destructive measurement was performed by Nakamura et al. ${ }^{6}$ to detect the state of the charge qubit. The qubit operation was performed at the charge degeneracy point, $u_{g}=1$, where the level splitting is minimal. An applied gate voltage then shifted the SCB working point (Fig. 5), inducing a large level splitting of the pure charge states $|0\rangle$ and $|1\rangle$ (the measurement preparation

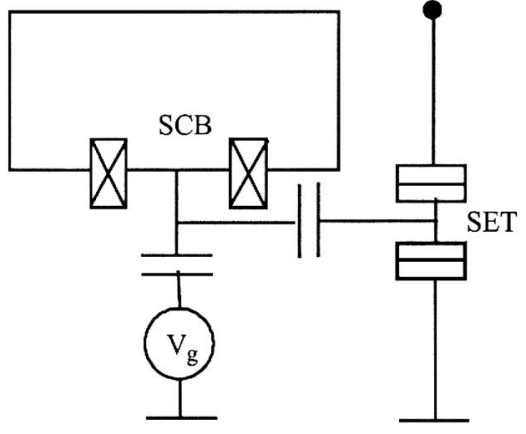

FIG. 10. Single electron transistor (SET) capacitively coupled to an SCB.

stage). In this process the upper $|1\rangle$ charge state went above the threshold for Cooper pair decay, creating two quasiparticles which immediately tunneled out via the probe junction into the leads. These quasiparticles were measured as a contribution to the classical charge current by repeating the experiment many times. Obviously, this type of measurement is also destructive.

\section{Measurement of charge qubit with SET}

Nondestructive measurement of the charge qubit has been implemented by connecting the qubit capacitively to a SET electrometer. ${ }^{103}$ The idea of this method is to use a qubit island as an additional SET gate (Fig. 10), controlling the dc current through the SET depending on the state of the qubit. When the measurement is to be performed, a driving voltage is applied to the SET, and the dc current is measured. Another version of the measurement procedure is to apply $\mathrm{rf}$ bias to the SET (rf-SET ${ }^{103-106}$ ) in Fig. 10, and to measure the dissipative or inductive response. In both cases the transmissivity will show two distinct values correlated with the two states of the qubit. Yet another version has recently been developed by the NEC group ${ }^{107}$ to perform single-shot readout: the Cooper pair on the SCB island then tunnels out onto a trap island (instead of the leads) used as a gate to control the current through the SET.

The physics of the SET-based readout has been extensively studied theoretically (see Refs. 44, 108, and 109 and references therein). A similar idea of controlling the transmission of a QPC (instead of an SET) capacitively coupled to a charge qubit has also been extensively discussed in the literature. ${ }^{110-114}$

The induced charge on the SET gate depends on the state of the qubit, affecting the SET working point and determining the conductivity and the average current. The development of the probability distributions of counted electrons with time is shown in Fig. 11.

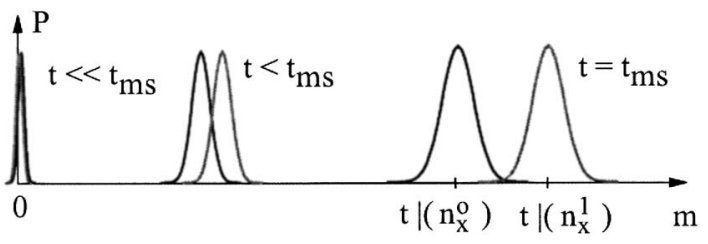

FIG. 11. Probability distributions $P$ of counted electrons as functions of time after the turning on of the measurement beam of electrons. Courtesy of G. Johansson, Chalmers. 
As the number of counted electrons grows, the distributions separate and become distinguishable, the distance between the peaks developing as $\sim N$ and the width as $\sim \sqrt{N}$. Detailed investigations ${ }^{114}$ show that the two electron-number probability distributions correlate with the probability of finding the qubit in either of two energy levels. The longtime development depends on the intensity and frequency distribution of the back-action noise from the electron current. With very weak detector back action, the qubit can relax to during the natural relaxation time $T_{1}$. With very strong back-action noise at the qubit frequency, the qubit may become saturated in a 50/50 mixed state.

\section{Measurement via coupled oscillator}

Another method of qubit readout that has attracted much attention concerns the measurement of the properties of a linear or nonlinear oscillator coupled to a qubit. This method is employed for the measurement of induced magnetic flux and persistent current in the loop of flux qubits and chargephase qubits, as well as for charge measurement on charge qubits. With this method, the qubit affects the characteristics of the coupled oscillator, e.g., changes the shape of the oscillator potential, after which the oscillator can be probed to detect the changes. There are two versions of the method: resonant spectroscopy of a linear tank circuit/cavity, and threshold detection using a biased JJ or SQUID magnetometer.

The first method uses the fact that the resonance frequency of a linear oscillator weakly coupled to the qubit undergoes a shift depending on the qubit state. The effect is most easily explained by considering the SCT Hamiltonian, Eq. (5),

$H_{S C T}=-\frac{1}{2}\left[\varepsilon \sigma_{z}+\Delta\left(\phi_{e}\right) \sigma_{x}\right]+\lambda\left(\phi_{e}\right) \widetilde{\phi} \sigma_{x}+4 E_{C} \widetilde{n}^{2}+\frac{1}{2} E_{L} \widetilde{\phi}^{2}$.

Let us proceed to the qubit energy basis, in which case the qubit Hamiltonian takes the form $-(E / 2) \sigma_{z}, E=\left(\varepsilon^{2}+\Delta^{2}\right)^{1 / 2}$. The interaction term in the qubit eigenbasis will consist of two parts, the longitudinal part, $\lambda_{z} \widetilde{\phi} \sigma_{z}, \lambda_{z}=(\Delta / E) \lambda$, and the transverse part, $\lambda_{x} \widetilde{\phi} \sigma_{x}, \lambda_{z}=(\varepsilon / E) \lambda$. In the limit of weak coupling the transverse part of interaction is the most essential. In the absence of interaction $\left(\phi_{e}=0\right)$ the energy spectrum of the qubit+oscillator system is

$$
E_{n \mp}= \pm \frac{E}{2}+\hbar \omega\left(n+\frac{1}{2}\right),
$$

where $\hbar \omega=\sqrt{8 E_{C} E_{L}}$ is the plasma frequency of the oscillator. The effect of weak coupling is enhanced in the vicinity of the resonance, when the oscillator plasma frequency is close to the qubit level spacing, $\hbar \omega \approx E$. Let us assume, however, that the coupling energy is smaller than the deviation from resonance, $\lambda_{x} \ll|\hbar \omega-E|$. Then the spectrum of the interacting system in the lowest perturbative order will acquire a shift,

$$
\delta E_{n \pm}= \pm(n+1) \frac{\lambda_{x}^{2} \hbar \omega}{E_{L}(\hbar \omega-E)} .
$$

This shift is proportional to the first power of the oscillator quantum number $n$, which implies that the oscillator

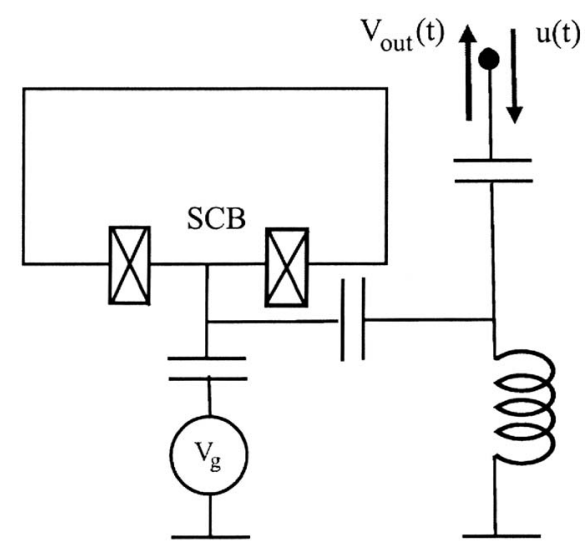

FIG. 12. SCT qubit coupled to a readout oscillator. The qubit is operated by input pulses $u(t)$. The readout oscillator is controlled and driven by ac microwave pulses $V_{g}(t)$. The output signal will be ac voltage pulses $V_{\text {out }}(t)$, the amplitude or phase of which may discriminate between the qubit " 0 " and " 1 " states.

frequency acquires a shift (the frequency of the qubit is also shifted $\left.^{115-119}\right)$. Since the sign of the oscillator frequency shift is different for the different qubit states, it is possible to distinguish the state of the qubit by probing this frequency shift.

In the case of the SCT, the LC oscillator is a generic part of the circuit. It is equally possible to use an additional LC oscillator inductively coupled to a qubit. This type of device has been described by Zorin ${ }^{120}$ for SCT readout, and was recently implemented for flux qubits by Il'ichev et al. ${ }^{33,41}$

Figure 12 illustrates another case, namely a charge qubit capacitively coupled to an oscillator, again providing energy resolution for discriminating the two qubit levels. ${ }^{121}$ Analysis of this circuit is similar that discussed below in the context of qubit coupling via oscillators, Section VII. The resulting Hamiltonian is similar to Eq. (50), namely,

$$
H=H_{S C B}+\lambda \sigma_{y} \phi+H_{\mathrm{osc}} .
$$

In comparison with the case of the SCT, Eq. (32) has a different form of the coupling term, which does not change during rotation to the qubit eigenbasis. Therefore the coupling constant $\lambda$ enters Eq. (31) directly. Recently, this type of read out has been implemented for a charge qubit by capacitively coupling the SCB of the qubit to a superconducting strip resonator. ${ }^{122-124}$

The measurement method described has turned out to be particularly useful for the charge qubits. The experimental data demonstrate the clear advantage of the degeneracy point, $n_{g}=1 / 2$, from the standpoint of decoherence: the coherence time drastically decreases with departure from this operating point, ${ }^{125}$ presumably due to fluctuating offset charges. On the other hand, the measurement of the charge at degeneracy is not efficient because the charge expectation values are the same for the both qubit states. The measurement via oscillator is efficient at degeneracy since it distinguishes the qubit energy levels. At small oscillator frequencies, the qubit adiabatically follows the oscillation of the gate voltage, and the qubit response can be expressed ${ }^{126,127}$ in terms of the second derivative of the qubit energy with respect to the gate voltage, $d^{2} E / d u_{g}^{2}$. The corresponding measured quantity can be thus interpreted as a quantum capaci- 


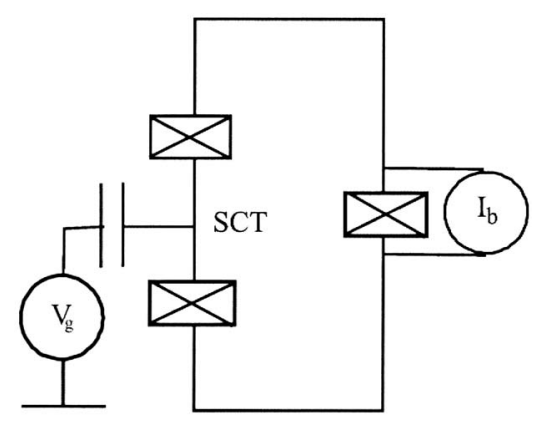

FIG. 13. SCT qubit coupled to a JJ readout quantum oscillator. The JJ oscillator is controlled by dc/ac current pulses $I_{b}(t)$ adding to the circulating currents in the loop due to the SCT qubit. The output will be dc/ac voltage pulses $V_{\text {out }}(t)$ discriminating between the qubit " 0 " and " 1 " states.

tance of the qubit. The measurement of the quantum capacitance was proven to be a quantum limited measurement. ${ }^{128}$

\section{Threshold detection}

To illustrate the threshold-detection method, let us consider an SCT qubit with a third Josephson junction inserted in the qubit loop, as shown in Fig. 13.

When the measurement of the qubit state is to be performed, a bias current is sent through the additional junction. This current is then added to the qubit-state dependent persistent current circulating in the qubit loop. If the qubit and readout currents flow in the same direction, the critical current of the readout $\mathrm{JJ}$ is exceeded, which induces the junction switching to the resistive branch, sending out a voltage pulse. This effect is used to distinguish the qubit states. The method has been extensively used experimentally by Vion et al. .2-29,129 $^{270}$

To describe the circuit, we add the Hamiltonian of a biased JJ, Eq. (1), to the SCT Hamiltonian (5). The phase quantization condition will now read: $2 \phi_{+}+\phi=\phi_{e}+\widetilde{\phi}$. The measurement junction will be assumed in the phase regime, $E_{J}^{m} \gg E_{C}^{m}$, and, moreover, the inductive energy will be the largest energy in the circuit, $E_{L} \gg E_{J}^{m}$. The latter implies that the induced phase is negligibly small and can be dropped from the phase quantization condition. We also assume that $\phi_{e}=0$, and thus $2 \phi_{+}+\phi=0$. Then, after having omitted the variable $\phi_{+}$, the kinetic energy term of the qubit can be combined with the much larger kinetic energy of the measurement junction, leading to insignificant renormalization of the measurement junction capacitance. As a result, the total Hamiltonian of the circuit will take the form

$$
\begin{aligned}
H= & E_{C}\left(n_{-}-n_{g}\right)^{2}-2 E_{J} \cos \left(\frac{\phi}{2}\right) \cos \phi_{-}+E_{C}^{m} n^{2} \\
& -E_{J}^{m} \cos \phi-\frac{\hbar}{2 e} I_{e} \phi .
\end{aligned}
$$

Since the measurement junction is supposed to be almost classical, its phase is fairly close to the minimum of the junction potential. During qubit operation, the bias current is zero; hence the phase of the measurement junction is zero. When the measurement is made, the current is ramped to a large value close to the critical current of the measurement junction, $I_{e}=(2 e / \hbar) E_{J}^{m}-\delta I$, tilting the junction potential and
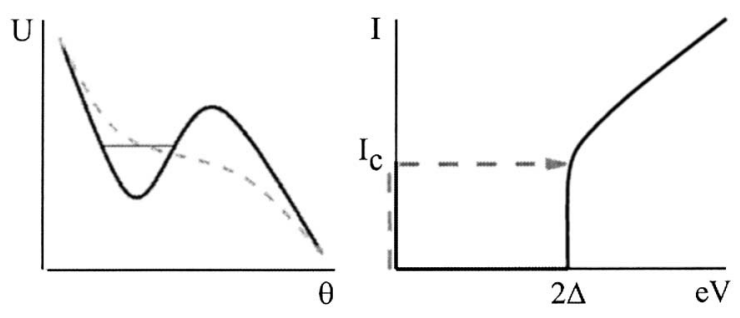

FIG. 14. Josephson potential energy of the measurement junction during the measurement (left): for the " 0 " qubit eigenstate there is a well (solid line) confining a level, while for the " 1 " qubit state there is no well (dashed line). Switching event on the current-voltage characteristic (right).

shifting the minimum towards $\pi / 2$. Introducing a new variable $\phi=\pi+\theta$, we expand the potential with respect to small $\theta \ll 1$ and, truncating the qubit part, we obtain

$$
H=-\frac{\varepsilon}{2} \sigma_{z}-\frac{\Delta}{2}\left(1-\frac{\theta}{2}\right) \sigma_{x}+E_{C}^{m} n^{2}-E_{J}^{m} \frac{\theta^{3}}{6}+\frac{\hbar}{2 e} \delta I \theta
$$

where $\Delta=2 \sqrt{E_{J}}$. The ramping is supposed to be adiabatic, so the phase remains at the minimum point. Let us analyze the behavior of the potential minimum by omitting a small kinetic term and diagonalizing the Hamiltonian (34). The corresponding eigenenergies depend on $\theta$,

$$
E_{ \pm}(\theta)=\mp \frac{E}{2}-E_{J}^{m} \frac{\theta^{3}}{6}+\left(\frac{\hbar}{2 e} \delta I \pm \frac{\Delta^{2}}{4 E}\right) \theta,
$$

as shown in Fig. 14. Then within the interval of bias currents $|\delta I| \leq-(2 e / \hbar)\left(\Delta^{2} / 4 E\right)$, the potential energy corresponding to the ground state has a local minimum, while for the excited state it does not. This implies that when the junction is in the ground state, no voltage will be generated. However, if the junction is in the excited state, it will switch to the resistive branch, generating a voltage pulse that can be detected.

In the setup discussed, the direction of the persistent current is measured. It is also possible to arrange the measurement of the flux by using a dc SQUID as a threshold detector. Such a setup is suitable for the measurement of flux qubits. Let us consider, for example, the three-junction flux qubit inductively coupled to a dc SQUID (Fig. 6). Then, under certain assumptions, the Hamiltonian of the system can be reduced to the following form:

$$
H=-\frac{1}{2}\left(\varepsilon \sigma_{z}+\Delta \sigma_{x}\right)+E_{C}^{s} n^{2}-\left(E_{J}^{s}+\lambda \sigma_{z}\right) \cos \phi-\frac{\hbar}{2 e} \delta I \phi,
$$

where $E_{J}^{s}$ is an effective (bias flux dependent) Josephson energy of the SQUID, and $\lambda$ is an effective coupling constant proportional to the mutual inductance of the qubit and the SQUID loops.

\section{EXPERIMENTS WITH SINGLE QUBITS AND READOUT DEVICES}

In this Section we shall describe a few experiments with single-qubits that represent the current state of the art and quite likely will be central components in the development of multi-qubit systems during the next five to ten years. The first experiment presents Rabi oscillations induced and observed in the elementary phase qubit and readout oscillator 
formed by a single JJ. ${ }^{14-16,35,36}$ The next example describes a series of recent experiments with a flux qubit ${ }^{30}$ coupled to different kinds of SQUID oscillator readout devices. ${ }^{31,32,130}$ A further example will discuss the charge-phase qubit coupled to a JJ-junction oscillator ${ }^{27}$ and the recent demonstration of extensive NMR-style operation of this qubit. ${ }^{29}$ The last example will present the case of a charge qubit (SCB) coupled to a microwave stripline oscillator, ${ }^{117,118,122,123}$ representing a solid-state analog of "cavity QED."

Before describing experiments and results, however, we will discuss in some detail the measurement procedures that give information about resonance line profiles, Rabi oscillations, and relaxation and decoherence times. The illustrations will be chosen from Vion et al. ${ }^{27}$ for the case of the chargephase qubit, but the examples are relevant for all types of qubits, representing fundamental procedures for studying quantum systems.

\section{A. Readout detectors}

Before discussing some of the actual experiments, it is convenient to describe some of basic readout-detector principles which more or less the same for the SET, rf-SET, JJ and SQUID devices. Atypical pulse scheme for exciting a qubit and reading out the response is shown in Fig. 5: the readout control pulse can be a dc pulse (DCP) or ac pulse (ACP). A DCP readout most often leads to an output voltage pulse, which may be quite destructive for the quantum system. An ACP readout presents a much weaker perturbation by probing the ac-response of an oscillator coupled to the qubit, creating much less back action, best representing QND readout.

\section{Spectroscopic detection of Rabi oscillation}

In the simplest use of the classical oscillator, it does not discriminate between the two different qubit states but only between energies of radiation emitted by a lossy resonator coupled to the qubit. In this way it is possible to detect the "low-frequency" Rabi oscillation of a qubit driven by continuous (i.e., not pulsed) high-frequency radiation tuned in the vicinity of the qubit transition energy. If the oscillator is tunable, the resonance window can be swept past the Rabi line. Alternatively, the Rabi frequency can be tuned and swept past the oscillator window by changing the qubit pumping power. $^{33}$

\section{Charge qubit energy level occupation from counting electrons: rf-SET}

In this case, the charge qubit is interacting with a beam of electrons passing through a single-electron transistor (SET) coupled to a charge qubit (e.g., the rf SET, ${ }^{103}$ ), as discussed in Section IV and illustrated in Fig. 10. In these cases the transmissivity of the electrons will show two distinct values correlated with the two states of the qubit.

\section{Coupled qubit-classical-oscillator system: switching detectors with dc-pulse output}

In Sec. IV we analyzed the case of an SCT qubit currentcoupled to a JJ-oscillator (Fig. 13) and discussed the Hamiltonian of the coupled qubit-JJ-oscillator system. The effect of the qubit was to deform the oscillator potential in different ways depending on the state of the qubit. The effect can then be probed in a number of ways, by input and output dc and ac voltage and current pulses, to determine the occupation of the qubit energy levels.

Using nonlinear oscillators like single JJs or SQUIDS one can achieve threshold and switching behavior where the JJ/SQUID switches out of the zero-voltage state, resulting in an output dc-voltage pulse.

\section{Switching JJ}

The method is based on the dependence of the critical current of the JJ on the state of the qubit, and consists of applying a short current DCP to the $\mathrm{JJ}$ at a value $I_{b}$ during a time $\Delta t$, so that the JJ will switch out of its zero-voltage state with a probability $P_{\mathrm{sw}}\left(I_{b}\right)$. For well-chosen parameters, the detection efficiency can approach unity. The switching probability then directly measures the qubit's energy level population.

\section{Switching SQUID}

In the experiments on flux qubits by the Delft group, two kinds of physical coupling of the SQUID to the qubit have been implemented, namely inductive coupling (Fig. 6 (left) $)^{12,130}$ and direct coupling (Fig. 6 (right)): ${ }^{30-32}$ The critical current of the SQUID depends on the flux threading the loop, and therefore is different for different qubit states. The problem is to detect a two percent variation in the SQUID critical current associated with a transition between the qubit states in a time shorter than the qubit energy relaxation time $T_{1}$. The SQUID behaves as an oscillator with a characteristic plasma frequency $\omega_{p}=\left[\left(L+L_{J}\right) C_{\mathrm{sh}}\right]^{-1 / 2}$. This frequency depends on the bias current $I_{b}$ and on the critical current $I_{C}$ via the Josephson inductance $L_{J}=\Phi_{0} / 2 \pi I_{C} \sqrt{1-I_{b}^{2} / I_{c}^{2}}$ (a shunt capacitor with capacitance $C_{\mathrm{sh}}$ and lead inductance $L$ is used to "tune" $\left.\omega_{p}\right)$. Thus the plasma frequency takes different values $\omega_{\pi}^{(0)}$ or $\omega_{\pi}^{(1)}$ depending on the state of the qubit, representing two different shapes of the SQUID oscillator potential.

In the dc-pulse-triggered switching SQUID, ${ }^{12,30,31}$ a dccurrent readout pulse is applied after the operation pulse(s) (Fig. 15), setting a switching threshold for the critical current. The circulating qubit current for one qubit state will then add to the critical current and make the SQUID switch

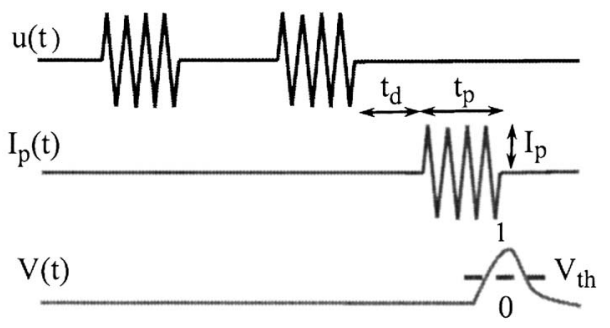

FIG. 15. Control pulse sequences involved in quantum state manipulations and measurement. Top: microwave voltage pulses $u(t)$ are applied to the control gate for state manipulation. Middle: a readout dc pulse (DCP) or ac pulse (ACP) $I_{b}(t)$ is applied to the threshold detector/discriminator a time $t_{d}$ after the last microwave pulse. Bottom: output signal $V(t)$ from the detector. The occurrence of a output pulse depends on the occupation probabilities of the energy eigenstates. A discriminator with threshold $V_{\text {th }}$ converts $V(t)$ into a boolean $0 / 1$ output for statistical analysis. 
$a$

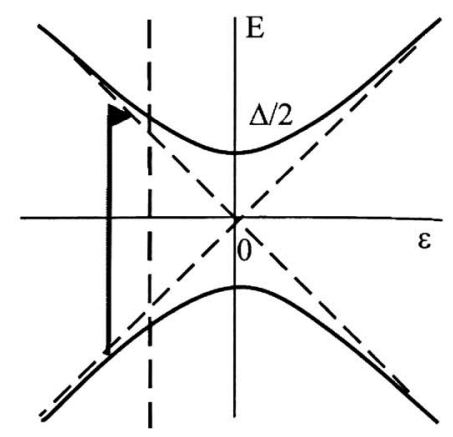

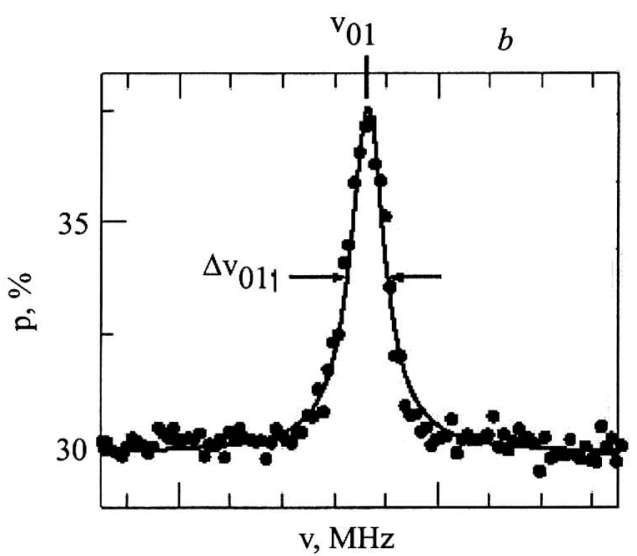

FIG. 16. Qubit energy level scheme. The qubit working point and transition energy is marked by the dashed line. The arrow marks the detuned microwave excitation $(a)$. Population of the upper level as a function of the detuning; the inverse of the half-width of the resonance line gives the total decoherence time $T_{2}(b)$.

to the voltage state, while the other qubit state will reduce the current and leave the SQUID in the zero-voltage state.

In an application of ac-pulse-triggered switching SQUID, ${ }^{32}$ readout relies on resonant activation by a microwave pulse at a frequency close to $\omega_{p}$, with the power adjusted so that the SQUID switches to the finite voltage state by resonant activation if the qubit is in state $|0\rangle$, whereas it stays in the zero-voltage state if it is in state $|1\rangle$. The resonant activation scheme is similar to the readout scheme used by Martinis et al. ${ }^{14,15,35,36}$

\section{Coupled qubit-classical-oscillator system: ac-pulse non- switching detectors}

This implementation of ACP readout uses the qubitSQUID combination ${ }^{12}$ shown in Fig. 6 (left), but with ACP instead of DCP readout, implementing a nondestructive dispersive method for the readout of the flux qubit. ${ }^{130}$ The detection is based on the measurement of the Josephson inductance of a dc SQUID inductively coupled to the qubit. Using this method, Lupascu et al. ${ }^{130}$ measured the spectrum of the qubit resonance line and obtained relaxation times around $80 \mu \mathrm{s}$, much longer than observed with DCP.

A related readout scheme was recently implemented by Siddiqi et al. ${ }^{131}$ using two different oscillation states of the nonlinear $\mathrm{JJ}$ in the zero-voltage state.

\section{B. Operation and measurement procedures}

A number of operation and readout pulses can be applied to a qubit circuit in order to measure various properties. The number of applied microwave pulses can vary depending on what quantities are to be measured: resonance line profile, relaxation time, Rabi oscillation, Ramsey interference, or spin echo, as discussed below.

\section{Resonance line profiles and $T_{2}$ decoherence times}

To study the resonance line profile, one applies a single long weak microwave pulse with given frequency, followed by a readout pulse (Fig. 16). The procedure is then repeated for a spectrum of frequencies. The Rabi oscillation amplitude, the upper state population, and the detector switching probability $p(t)$ will depend on the detuning and will grow towards resonance. The linewidth gives directly the total in- verse decoherence lifetime $1 / T_{2}=1 / 2 T_{1}+1 / T_{\phi}$. The decoherence-time contributions from relaxation $\left(1 / T_{1}\right)$ and dephasing $\left(1 / T_{\phi}\right)$ can be (approximately) separately measured, as discussed below.

\section{$T_{1}$ relaxation times}

To determine the $T_{1}$ relaxation time one measures the decay of the population of the upper $|1\rangle$ state after a long microwave pulse saturating the transition, varying the delay time $t_{d}$ of the detector readout pulse (Figs. 17 and 18). The measured $T_{1}=1.8$ microseconds is so far the best value for the quantronium charge-phase qubit.

\section{Rabi oscillations and $T_{2, \text { Rabi }}$ decoherence time}

To study Rabi oscillations (frequency $\Omega-u$, the amplitude of driving field) one turns on a resonant microwave pulse for a given time $t_{\mu \mathrm{w}}$ and measures the upper $|1\rangle$ state population (probability) $p_{1}(t)$ after a given (short) delay time $t_{d}$. If the systems is perfectly coherent, the state vector will develop as $\cos \Omega t|0\rangle+\sin \Omega t|1\rangle$, and the population of the upper state will then oscillate as $\sin ^{2} \Omega t$ between 0 and 1 . In the presence of decoherence, the amplitude of the oscillation of $p_{1}(t)$ will decay on a time scale $T_{\text {Rabi }}$ towards the average value $p_{1}(t=\infty)=0.5$. This corresponds to incoherent saturation of the 0 to 1 transition.

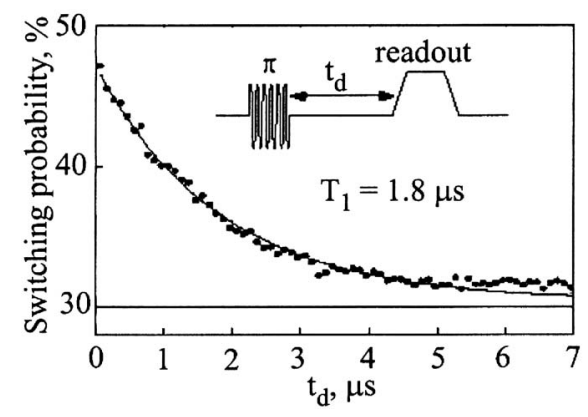

FIG. 17. Decay of the switching probability of the charge-qubit readout junction as a function of the delay time $t_{d}$ between the excitation and readout pulses. Courtesy of D. Esteve, CEA-Saclay. 


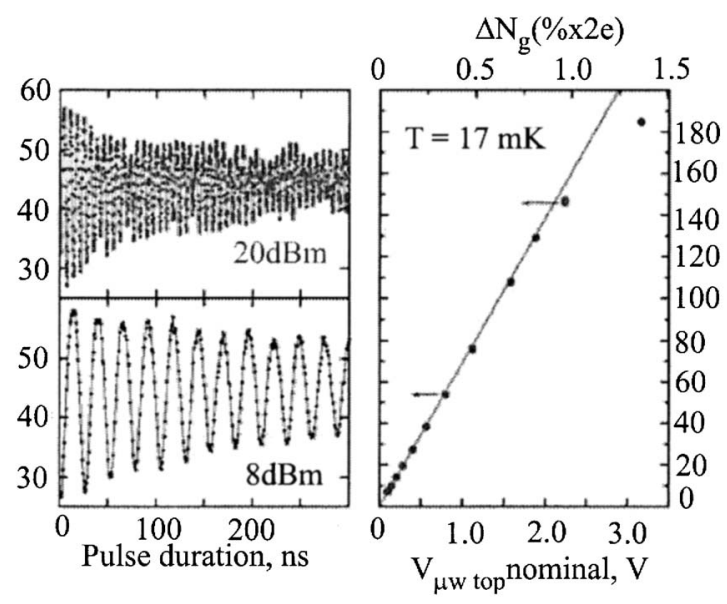

FIG. 18. Rabi oscillations of the switching probability measured just after a resonant microwave pulse of duration (left); measured Rabi frequency (dots) varies linearly with microwave amplitude (voltage) as expected (right). Courtesy of D. Esteve, CEA-Saclay.

\section{Ramsey interference, dephasing and $T_{2, \text { Ramsey }}$ decoherence time}

The Ramsey interference experiment measures the decoherence time of the non-driven, freely precessing, qubit. In this experiment a $\pi / 2$ microwave pulse around the $x$ axis induces Rabi oscillation that tips the spin from the north pole down to the equator. The spin vector rotates in the $x y$ plane, and after a given time $\Delta t$, another $\pi / 2$ microwave pulse is applied, immediately followed by a readout pulse (Fig. 19).

Since the $\pi / 2$ pulses are detuned by $\delta$ from the qubit $|0\rangle \rightarrow|1\rangle$ transition frequency, the qubit will precess with frequency $\delta$ relative to the rotating frame of the driving field. Since the second microwave pulse will be applied in the plane of the rotating frame, it will have a projection $\cos \delta t$ on the qubit vector and will drive the qubit towards the north or south poles, resulting in a specific time-independent final superposition state $\cos \delta t|0\rangle+\sin \delta t|1\rangle$ of the qubit at the end of the last $\pi / 2$ pulse. The readout pulse then catches the qubit in this superposition state and forces it to decay if the qubit is in the upper $|1\rangle$ state. The probability will oscillate with the detuning frequency, and a single-shot experiment will then detect the upper state with this probability. Repeat-

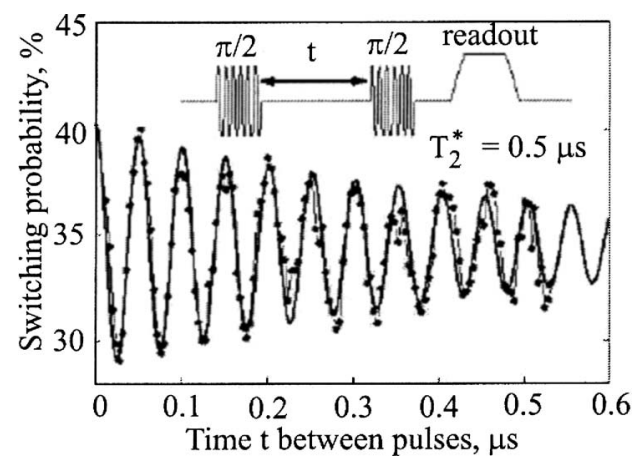

FIG. 19. Ramsey fringes of the switching probability after two phasecoherent microwave $\pi / 2$ pulses separated by the time delay $t$. The continuous line represents a fit by exponentially damped cosine function with time constant $T_{2}^{*}=T_{\phi}=0.5 \mu$ s. The oscillation period coincides with the inverse of the detuning frequency (here $\nu-\nu_{01}=20.6 \mathrm{MHz}$ ). Courtesy of D. Esteve, CEA-Saclay. ing the experiment many times for different $\pi / 2$ pulse separation $\Delta t$ will then give $|0\rangle$ or $|1\rangle$ with probabilities $\cos ^{2} \delta t$ and $\sin ^{2} \delta t$. By taking the average and then varying the pulse separation, one will trace out the Ramsey interference oscillatory signal. Dephasing will make the signa decay on the time scale $T_{\phi}$.

\section{Spin-echo}

The spin-echo and Ramsey pulse sequences differ in that a $\pi$ pulse around the $x$ axis is added in between the two $\pi / 2$ pulses in the spin-echo experiment. As in the Ramsey experiment, the first $\pi / 2$ pulse makes the Bloch vector start rotating in the equatorial $x y$ plane with frequency $E / \hbar=\nu_{01}$. The effect of the $\pi$ pulse is now to flip the entire $x y$ plane with the rotating Bloch vector around the $x$ axis, reflecting the Boch vector in the $x z$ plane. The Bloch vector then continues to rotate in the $x y$ plane in the same direction. Finally a second $\pi / 2$ pulse is applied to project the state on the $z$ axis.

If two Bloch vectors with slightly different frequency start rotating at the same time in the $x y$ plane, they will move with different angular speeds. The effect of the $\pi$ pulse at time $\Delta t$ will be to permute the Bloch vectors, and then let the motion continue in the same direction. This is similar to reversing the motion and letting the Bloch vectors backtrace. The net result is that the two Bloch vectors re-align after time $2 \Delta t$.

In NMR experiments, the different Bloch vectors correspond to different spins in the ensemble. In the case of a single qubit, the implication is that in aseries of repeated experiments, the result will be insensitive to small variations $\delta E$ of the qubit energy between measurements, as long as the energy (rotation frequency) is constant during one and the same measurement. If fluctuations occur during one measurement, then this cannot be corrected for. The spin-echo procedure can therefore remove the measurement-related line-broadening associated with slow fluctuations of the qubit precession and allow observation of the intrinsic coherence time of the qubit.

\section{PHYSICAL COUPLING SCHEMES FOR TWO QUBITS}

\section{A. General principles}

A generic scheme for coupling qubits is based on the physical interaction of linear and nonlinear oscillators constituting a superconducting circuit. In a multi-qubit system the induced gate charge in the SCB, or the flux through the SQUID loop, or the phase in the Josephson energy, will be a sum of contributions from several (in principle, all) qubits. The energy of the system therefore cannot be described as the sum of two independent qubits because of the quadratic dependence, and the cross terms represent interaction energies of different kinds: capacitive, inductive, and phase/ current.

Moreover, using JJ circuits as nonlinear coupling elements we have the advantage that the direct physical coupling strength may be controlled, e.g., by tuning the inductance via current-biased JJs, or tuning the capacitance by a voltage-biased SCB. 


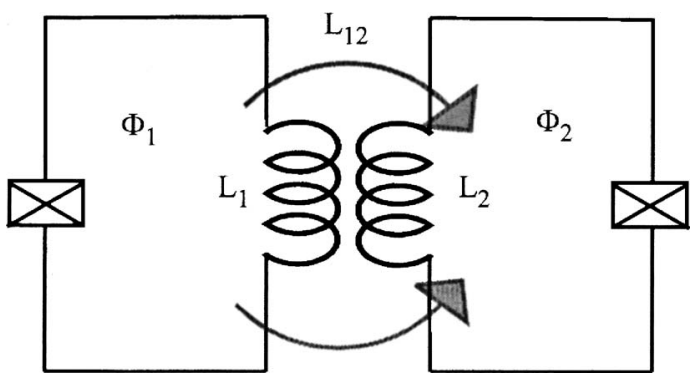

FIG. 20. Fixed inductive (flux) coupling of elementary flux qubit. The loops can be separate, or have a common leg like in the figure.

\section{Inductive coupling of flux qubits}

A common way of coupling flux qubits is the inductive coupling: magnetic flux induced by one qubit threads the loop of another qubit, changing the effective external flux (Fig. 20). This effect is taken into account by introducing the inductance matrix $L_{i k}$, which connects flux in the $i$ th loop with the current circulating in the $k$ th loop:

$$
\phi_{i}=\sum_{k} L_{i k} I_{k} .
$$

The off-diagonal element of this matrix, $L_{12}$, is the mutual inductance, which is responsible for the interaction. By using the inductance matrix, the magnetic part of the potential energy in Eq. (2) can be generalized to the case of two coupled qubits,

$$
\frac{1}{2}\left(\frac{\hbar}{2 e}\right)^{2} \sum_{i k}\left(L^{-1}\right)_{i k}\left(\phi-\phi_{e i}\right)\left(\phi_{k}-\phi_{e k}\right) .
$$

Then, following the truncation procedure leading to the flux qubit, we calculate the matrix elements,

$$
\langle l|\widetilde{\phi}-f| l\rangle, \quad\langle r|\widetilde{\phi}-f| r\rangle, \quad\langle l|\phi-f| r\rangle,
$$

for each qubit. The last matrix element is exponentially small, while the first two are approximately equal to the minimum points of the potential energy, $\phi_{l}$ and $\phi_{r}$, respectively. This implies that the truncated interaction basically has the $z z$ form,

$$
\begin{aligned}
& H_{\mathrm{int}}=\lambda \sigma_{z 1} \sigma_{z 2}, \\
& \lambda=\frac{1}{8}\left(\frac{\hbar}{2 e}\right)^{2}\left(L^{-1}\right)_{12}\left(\phi_{l}-\phi_{r}\right)_{1}\left(\phi_{l}-\phi_{r}\right)_{2} .
\end{aligned}
$$

\section{Capacitive coupling of charge qubits}

One of the simplest coupling schemes is the capacitive coupling of charge qubits. Such a coupling is realized by connecting the islands of two SCBs via a small capacitor, as illustrated in Fig. 21.

This will introduce an additional term in the Lagrangian of the two noninteracting SCBs, namely the charging energy $C_{3}$ of the capacitor, $\delta L=C_{3} V_{3}^{2} / 2$. The voltage drop $V_{3}$ across the capacitor is expressed via the phase differences across the qubit junctions, $V_{3}=(\hbar / 2 e)\left(\dot{\phi}_{1}-\dot{\phi}_{2}\right)$, and thus the kinetic part of the Lagrangian will take the form

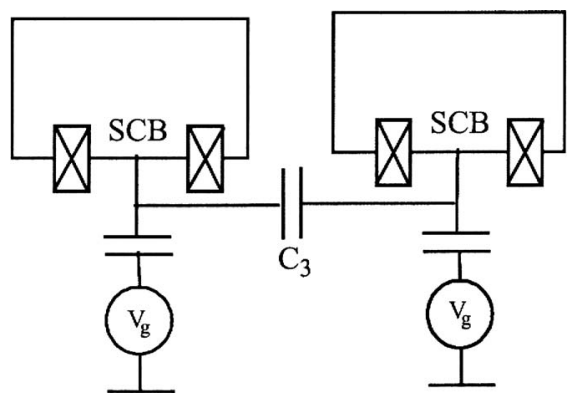

FIG. 21. Fixed capacitive coupling of charge qubits.

$$
K\left(\dot{\phi}_{1}, \dot{\phi}_{2}\right)=\frac{1}{2}\left(\frac{\hbar}{2 e}\right)^{2} \sum_{i, k} C_{i k} \dot{\phi}_{i} \dot{\phi}_{k}-\frac{\hbar}{2 e} \sum_{i}^{2} C_{g i} V_{g i} \dot{\phi}_{i},
$$

where the capacitance matrix elements are $C_{i i}=C_{\Sigma_{i}}+C_{3}$, and $C_{12}=C_{3}$. Then proceeding to the circuit Hamiltonian, we find the interaction term,

$$
H_{\text {int }}=2 e^{2}\left(C^{-1}\right)_{12} n_{1} n_{2} \text {. }
$$

This interaction term is diagonal in the charge basis, and therefore leads to the $z z$ interaction after truncation,

$$
H_{\text {int }}=\lambda \sigma_{z 1} \sigma_{z 2}, \quad \lambda=\frac{e^{2}}{2}\left(C^{-1}\right)_{12} .
$$

The qubit Hamiltonians are given by Eq. (19) with charging energies renormalized by the coupling capacitor.

\section{$J$ phase coupling of charge qubits}

Instead of the capacitor, the charge qubits can be connected via a Josephson junction. ${ }^{132}$ In this case, the Josephson energy of the coupling junction $E_{J 3} \cos \left(\phi_{1}-\phi_{2}\right)$ must be added to the Lagrangian in addition to the charging energy. This interaction term is apparently off-diagonal in the charge basis and, after truncation, gives rise to the so-called $x y$ coupling,

$$
H_{\mathrm{int}}=\lambda\left(\sigma_{x 1} \sigma_{x 2}+\sigma_{y 1} \sigma_{y 2}\right), \quad \lambda=\frac{E_{J 3}}{4} .
$$

\section{Capacitive coupling of single JJs}

Capacitive coupling of JJ qubits, illustrated in Fig. 22, is

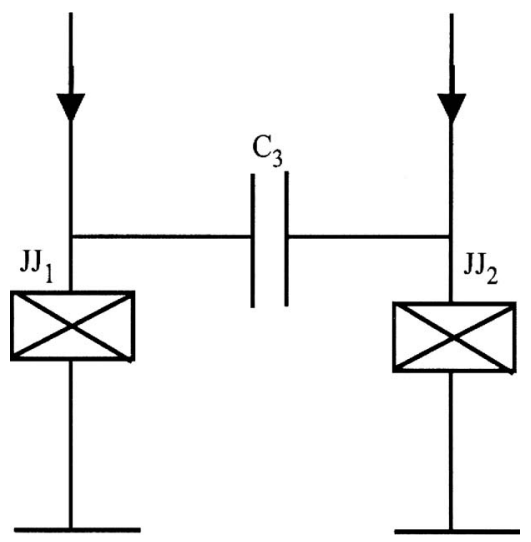

FIG. 22. Capacitive coupling of single JJ qubits. 


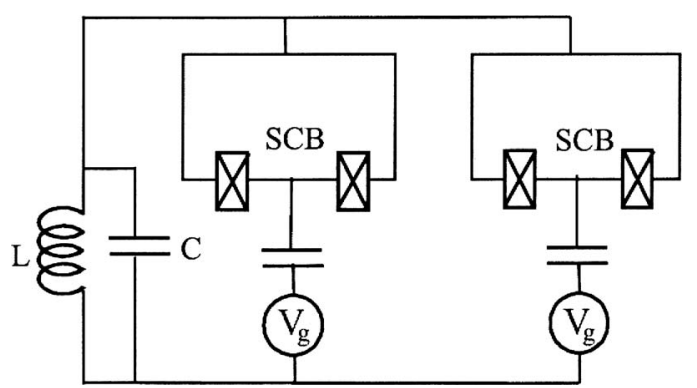

FIG. 23. Two charge qubits coupled to a common $L C$ oscillator.

described in a way similar to the charge qubit, and the resulting interaction Hamiltonian has the form given in Eq. (42).

Generally, in the qubit eigenbasis, $|0\rangle$ and $|1\rangle$, all matrix elements of the interaction Hamiltonian are nonzero. However, if we adopt a parabolic approximation for the Josephson potential, then the diagonal matrix elements turn to zero, $n_{00}=n_{11}=0$, while the off-diagonal matrix elements remain finite, $n_{01}=-n_{10}=-i\left(E_{J} / E_{C}\right)^{1 / 4}$. Then, after truncation, the charge number operator $n$ turns to $\sigma_{y}$, and the qubit-qubit interaction takes the $y y$ form,

$$
H_{\mathrm{int}}=\lambda \sigma_{y 1} \sigma_{y 2}, \quad \lambda=2 e^{2} \sqrt{\frac{\hbar^{2} \omega_{p 1} \omega_{p 2}}{E_{C 1} E_{C 2}}}\left(C^{-1}\right)_{12} .
$$

\section{B. Coupling via oscillators}

Besides the direct coupling schemes described above, several schemes of coupling qubits via auxiliary oscillators have been considered. ${ }^{44}$ Such schemes provide more flexibility, e.g., to control qubit interaction, to couple two remote qubits, and to connect several qubits. Moreover, in many advanced qubits, the qubit variables are generically connected to the outside world via an oscillator (e.g., the Delft and Saclay qubits). To explain the principles of such a coupling, we consider the coupling scheme for charge qubits suggested by Shnirman $e t$ al. ${ }^{21}$

\section{Coupling of charge (SCB, SCT) qubits}

In this circuit the island of each SCB is connected to ground via a common $L C$ oscillator, as illustrated in Fig. 23. The kinetic energy of a single qubit should now be modified to take into account the additional phase difference $\phi$ across the oscillator,

$$
K\left(\dot{\phi}_{-, i}, \dot{\phi}\right)=\frac{1}{2}\left(\frac{\hbar}{2 e}\right)^{2}\left[2 C \phi_{-, i}^{2}+C_{g}\left(V_{g i}-\dot{\phi}-\dot{\phi}_{-, i}\right)^{2}\right] .
$$

The cross term in this equation can be made to vanish by a change of qubit variable,

$$
\phi_{-, i}=\phi_{i}-a \phi, \quad a=\frac{C_{g}}{C_{\Sigma}} .
$$

The kinetic energy will then split into two independent parts, the kinetic energy of the qubit, and an additional quadratic term,

$$
\frac{1}{2}\left(\frac{\hbar}{2 e}\right)^{2} \frac{C C_{g}}{C_{\Sigma}} \dot{\phi}^{2}
$$

which should be combined with the kinetic energy of the oscillator, leading to renormalization of oscillator capacitance.

Expanding the Josephson energy, after the change of variable, gives

$$
E_{J i} \cos \left(\phi_{i}-a \phi\right) \approx E_{J i} \cos \phi_{i}-E_{J i} a \phi \sin \phi_{i} .
$$

provided the amplitude of the oscillations of $\phi$ is small. The last term in this equation describes the linear coupling of the qubit to the $L C$ oscillator.

Collecting all the terms in the Lagrangian and performing quantization and truncation procedures, we arrive at the following Hamiltonian of the qubits coupled to the oscillator (this is similar to Eq. (32) for the SCT),

$$
H=\sum_{i=1,2}\left(H_{S C B, i}+\lambda_{i} \sigma_{y i} \phi\right)+H_{\mathrm{osc}},
$$

where $H_{S C B}^{(i)}$ is given by Eq. (19), and

$$
\lambda_{i}=\frac{E_{J i} C_{g}}{C_{\Sigma}},
$$

is the coupling strength.

The physics of the qubit coupling in this scheme is the following: quantum fluctuation of the charge of one qubit produces a displacement of the oscillator, which perturbs the other qubit. If the plasma frequency of the $L C$ oscillator is much larger than the frequencies of all qubits, then virtual excitation of the oscillator will produce a direct effective qubit-qubit coupling, the oscillator staying in the ground state during all qubit operations. To provide a small amplitude of the zero-point fluctuations, the oscillator plasma frequency should be small compared to the inductive energy, or $E_{C o s c} \ll E_{L}$. Then the fast fluctuations can be averaged out. Noticing that the displacement does not change the oscillator ground-state energy, which then drops out after the averaging, we finally arrive at the Hamiltonian of the direct effective qubit coupling,

$$
H_{\mathrm{int}}=-\frac{\lambda_{1} \lambda_{2}}{E_{L}} \sigma_{y 1} \sigma_{y 2}
$$

for the oscillator-coupled charge qubits in Fig. 23.

\section{Current coupling of SCT qubits}

Charge qubits based on SCTs can be coupled by connecting loops of neighboring qubits by a large Josephson junction in the common link, ${ }^{133-139}$ as illustrated in Fig. 24.

The idea is similar to the previous one: to couple qubit variables to a new variable, the phase of the coupling Josephson junction, then to arrange the phase regime for the junction with large plasma frequency $\left(E_{C \text { coupl }} \ll E_{\text {Jcoupl }}\right)$, and then to average out the additional phase. Technically, the circuit is described using the SCT Hamiltonian, Eqs. (5) and (21), for each qubit, 


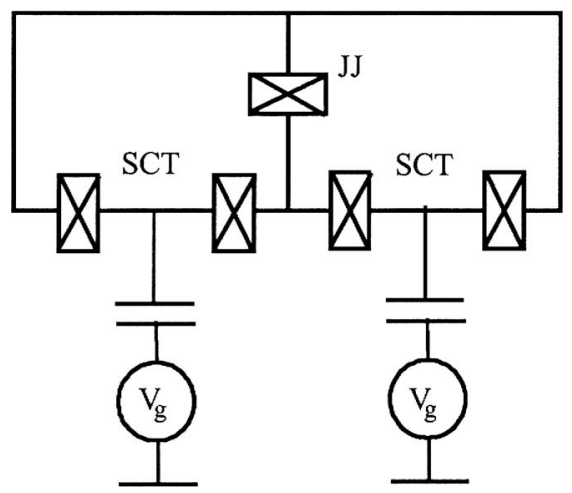

FIG. 24. Charge (charge-phase) qubits coupled via a common Josephson junction providing phase coupling of the two circuits.

$$
\begin{aligned}
H_{S C T}= & E_{C}\left(n_{-}-n_{g}\right)^{2}+E_{C} n_{+}^{2}-2 E_{J} \cos \phi+\cos \phi_{-} \\
& +E_{L} \frac{\left(2 \phi_{+}-\phi_{e}\right)^{2}}{2},
\end{aligned}
$$

and adding the Hamiltonian of the coupling junction,

$$
H_{c}=E_{C, c} n_{c}^{2}-E_{J, c} \cos \phi_{c} .
$$

The phase $\phi_{c}$ across the coupling junction must be added to the flux quantization condition in each qubit loop; e.g., for the first qubit $2 \phi_{+, 1}+\phi_{c}=\phi_{e, 1}+\widetilde{\phi}_{1}$ (for the second qubit the sign of $\phi_{c}$ will be negative). Assuming small inductive energy, $E_{L} \ll E_{J, c}$, we may neglect $\tilde{\phi}$; then, assuming the flux regime for the coupling Josephson junction, we adopt a parabolic approximation for the junction potential, $E_{J, c} \phi_{c}^{2} / 2$.

With these approximations, the Hamiltonian of the first qubit plus coupling junction will take a form similar to Eq. (53), where $E_{J, c}$ will substitute for $E_{L}$ and $\phi_{c}$ will substitute for $2 \phi_{+}-\phi_{e}$. Finally, assuming the amplitude of the $\phi_{c}$ oscillations to be small, we proceed as in the previous subsection, i.e., expand the cosine term, obtaining a linear coupling between the SCB and the oscillator, truncate the full Hamiltonian, and average out the oscillator. This will yield the following interaction term:

$$
H_{\mathrm{int}}=\frac{\lambda_{1} \lambda_{2}}{E_{J, c}} \sigma_{x 1} \sigma_{x 2}, \quad \lambda_{i}=E_{J} \sin \frac{\phi_{i}}{2} .
$$

This coupling scheme also applies to flux qubits: in this case, the coupling will have the same form as in Eq. (40), but the strength will be determined by the Josephson energy of the coupling junction, cf. Eq. (55), rather than by the mutual inductance.

\section{Variable coupling schemes}

Computing with quantum gate networks basically assumes that one- and two-qubit gates can be turned on and off at will. This can be achieved by tuning qubits with fixed, finite coupling in and out of resonance, in NMR-style computing. ${ }^{140}$

Here we shall discuss an alternative way, namely to vary the strength of the physical coupling between nearestneighbor qubits, as discussed in a number of recent papers. ${ }^{133,134,136-138,141-144}$

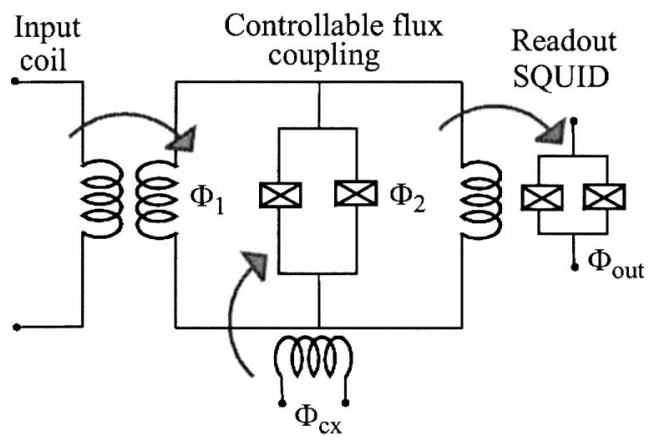

FIG. 25. Flux transformer with variable coupling controlled by a SQUID.

\section{Variable inductive coupling}

To achieve variable inductive coupling of flux qubits one has to be able to control the mutual inductance of the qubit loops. This can be done by different kinds of controllable switches (SQUIDS, transistors) ${ }^{141}$ in the circuit. In a recent experiment, a variable flux transformer was implemented as a coupling element (see Fig. 25) by controlling the transforming ratio. ${ }^{145}$ The flux transformer is a superconducting loop strongly inductively coupled to the qubit loops, which are distant from each other so that the direct mutual qubit inductance is negligibly small. Because of the effect of quantization of magnetic flux in the transformer loop, ${ }^{146}$ a local variation of the magnetic flux $\Phi_{1}$ induced by one qubit will affect the local magnetic flux $\Phi_{2}$ in the vicinity of the other qubit, creating effective qubit-qubit coupling. When a dc SQUID is inserted in the transformer loop, as shown in Fig. 25 , it will short-circuit the transformer loop, and the transformer ratio $\Phi_{2} / \Phi_{1}$ will change. The effect depends on the current flowing through the SQUID and is proportional to the critical current of the SQUID. The latter is controlled by applying a magnetic flux $\Phi_{c x}$ to the SQUID loop, as shown in Fig. 25. Quantitatively, the dependence of the transformer ratio on the controlling flux is given by the equation ${ }^{145}$

$$
\frac{\Phi_{2}}{\Phi_{1}}=\left(1+\frac{E_{J}}{E_{L}} \cos \frac{\pi \Phi_{c x}}{\Phi_{0}}\right)^{-1},
$$

where $E_{J}$ is the Josephson energy of the SQUID junction, and $E_{L}$ is the inductive energy of the transformer.

\section{Variable Josephson coupling}

A variable Josephson coupling is obtained when a single Josephson junction is substituted by a symmetric dc SQUID whose effective Josephson energy $2 E_{J} \cos \left(\phi_{e} / 2\right)$ depends on the magnetic flux threading the SQUID loop. This property is commonly used to control level spacing in both flux and charge qubits, and it can also be used to switch qubit-qubit couplings on and off. For example, the coupling of the charge-phase qubits via the Josephson junction in Fig. 24 can be made variable by substituting the single coupling junction with a de SQUID. ${ }^{133,134}$

The coupling scheme shown in Fig. 23 is made controllable by using a dc SQUID design for the SCB. Indeed, since the coupling strength depends on the Josephson energy of the qubit junction, Eq. (51), this solution provides variable coupling of the qubits. Similarly, the coupling of the SCTs shown in Fig. 24 can be made controllable by employing a 


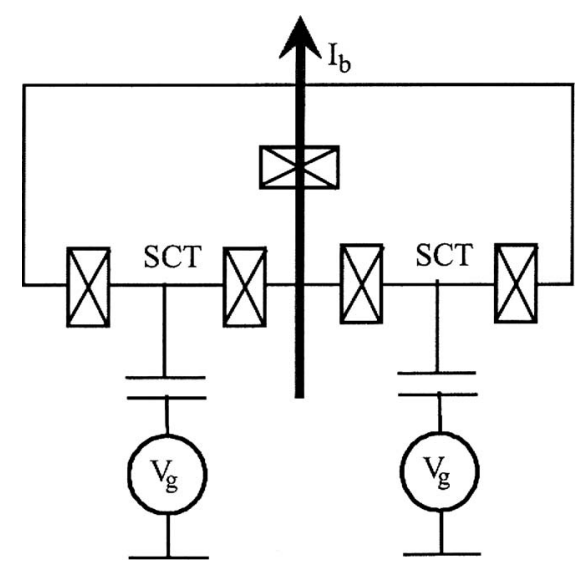

FIG. 26. Coupled charge qubits with current-controlled phase coupling: the arrow indicates the direction of the controlling bias current.

dc SQUID as a coupling element. A disadvantage of this solution is that the qubit parameters will vary simultaneously with varying of the coupling strength. A more general drawback of the dc SQUID-based controllable coupling is the necessity of applying magnetic field locally, which might be difficult to achieve without disturbing other elements of the circuit. This is, however, an experimental question, and what are practical solutions in the long run remains to be seen.

\section{Variable phase coupling}

An alternative solution for varying the coupling is based on the idea of controlling the properties of the Josephson junction by applying external dc current, ${ }^{136-138}$ as illustrated in Fig. 26. The coupling strength here depends on the plasma frequency of the coupling Josephson junction, which in turn depends on the form of the local minimum of the junction potential energy. This form can be changed by tilting the junction potential by applying external bias current. The role of the external phase bias, $\phi_{e}$, will now be played by the minimum point $\phi_{0}$ of the tilted potential determined by the applied bias current, $E_{J, c} \sin \phi_{0}=(\hbar / 2 e) I_{e}$. Then the interaction term will read,

$$
H_{\text {int }}=\lambda \sigma_{x 1} \sigma_{x 2}, \quad \lambda=\frac{E_{J}^{2} \sin ^{2}\left(\phi_{0} / 2\right)}{E_{J, c} \cos \phi_{0}},
$$

and local magnetic field biasing is not required.

\section{Variable capacitive coupling}

Variable capacitive coupling of charge qubits based on a quite different physical mechanism of interacting SCB charges has been proposed in Ref. 143. The SCBs are then connected via the circuit presented in Fig. 27.

The Hamiltonian of this circuit, including the charge qubits, has the form

$$
H=\sum_{i} H_{S C B, i}+E_{C}\left(n-q\left(n_{1}+n_{2}\right)\right)^{2}-E_{J} \cos \phi,
$$

where $E_{C}$ and $E_{J} \sim E_{c}$ are the charging and Josephson energies of the coupling junction, and $n$ and $\phi$ are the charge and the phase of the coupling junction. The function $q$ is a linear function of the qubit charges, $n_{1}$ and $n_{2}$, and it also depends on the gate voltages of the qubits and the coupling junction.

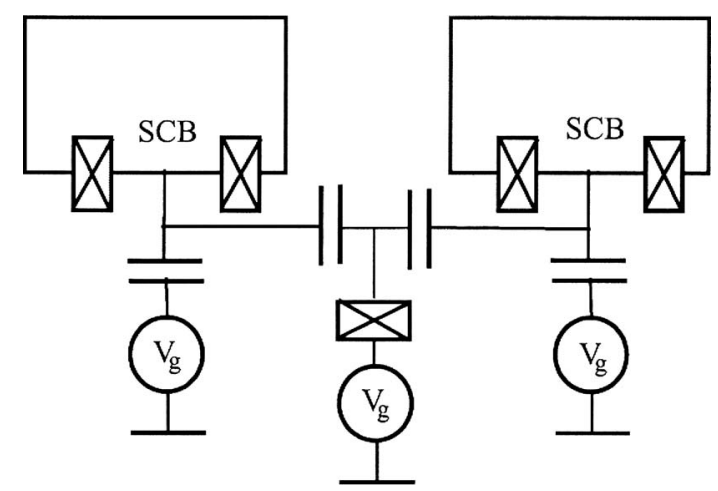

FIG. 27. Variable capacitance tuned by a voltage-controlled SCB.

In contrast to the previous scheme, here the coupling junction is not assumed to be in the phase regime; however, it is still supposed to be fast, $E_{J} \gg E_{J i}$. Then the energy gap in the spectrum of the coupling junction is much bigger than the qubit energy, and the junction will stay in the ground state during qubit operations. Then after truncation, and averaging out the coupling junction, the Hamiltonian of the circuit will take the form

$$
H=\sum_{i} H_{S C B, i}+\varepsilon_{0}\left(\sigma_{z 1}+\sigma_{z 2}\right),
$$

where the qubit Hamiltonian is given by Eq. (19), and the function $\varepsilon_{0}$ is the ground-state energy of the coupling junction. The latter can generally be presented as a linear combination of terms proportional to $\sigma_{z 1} \sigma_{z 2}$ and $\sigma_{z 1}+\sigma_{z 2}$,

$$
\varepsilon_{0}\left(\sigma_{z 1}+\sigma_{z 2}\right)=\alpha+\nu \sigma_{z 1} \sigma_{z 2}+\beta\left(\sigma_{z 1}+\sigma_{z 2}\right),
$$

with coefficients depending on the gate potentials. The second term in this expression gives the $z z$ coupling (in the charge basis), and the coupling constant $\nu$ may, according to the analysis of Ref. 143, take on both positive and negative values, depending on the coupling junction gate voltage. In particular, it may turn to zero, implying qubit decoupling.

\section{Two qubits coupled via a resonator}

In the previous discussion, the coupling oscillator plays a passive role, being enslaved by the qubit dynamics. However, if the oscillator is tuned into resonance with a qubit, then the oscillator dynamics will become essential, leading to qubit-oscillator entanglement. In this case, the approximation of direct qubit-qubit coupling is not appropriate; instead, manipulations explicitly involving the oscillator must be considered.

Let us consider, as an example, operations with two charge qubits capacitively coupled to the oscillator. Assuming the qubits to be biased at the degeneracy point and proceeding to the qubit eigenbasis (phase basis in this case), we write the Hamiltonian in the form

$$
H=-\sum\left(\frac{\Delta_{i}}{2} \sigma_{z i}-\lambda_{i} \sigma_{x} \phi_{i}\right)+H_{\text {osc }}[\phi] .
$$

Let us consider the following manipulation involving the variation of the oscillator frequency: ${ }^{142}$ at time $t=0$, the oscillator frequency is off-resonance with both qubits, $\hbar \omega(0)$ $<\Delta_{1}<\Delta_{2}$. Then the frequency is rapidly ramped so that the 
oscillator becomes resonant with the first qubit, $\hbar \omega\left(t_{1}\right)=\Delta_{1}$, the frequency remaining constant for a while. Then the frequency is ramped again and brought into resonance with the second qubit, $\hbar \omega\left(t_{2}\right)=\Delta_{2}$. Finally, after a certain time it is ramped further so that the oscillator gets out of resonance with both qubits at the end, $\hbar \omega\left(t>t_{3}\right)>\Delta_{2}$.

When passing through the resonance, the oscillator is hybridized with the corresponding qubit, and after passing the resonance, the oscillator and qubit have become entangled. For example, let us prepare our system at $t=0$ in the excited state $\psi(0)=|100\rangle=|1\rangle|0\rangle|0\rangle$, where the first number denotes the state of the oscillator (first excited level), and the last numbers denote the (ground) states of the first and second qubits, respectively. After the first operation, the oscillator will be entangled with the first qubit, $\psi\left(t_{1}<t<t_{2}\right)$ $=\left(\cos \theta_{1}|10\rangle+\sin \theta_{1} e^{i \alpha}|01\rangle\right)|0\rangle$. After the second manipulation, the state $|100\rangle$ will be entangled with state $|001\rangle$,

$$
\begin{gathered}
\psi\left(t>t_{3}\right)=\cos \theta_{1}\left(\cos \theta_{2}|100\rangle+\sin \theta_{2} \mathrm{e}^{i \beta}|001\rangle\right) \\
+\sin \theta_{1} \mathrm{e}^{i \alpha}|010\rangle .
\end{gathered}
$$

To ensure that there are no more resonances during the manipulations described, it is sufficient to require $\hbar \omega(0)>\Delta_{2}$ $-\Delta_{1}$.

If the controlling pulses are chosen so that $\theta_{2}=\pi / 2$, then the initial excited state will be eliminated form the final superposition, and we'll get entangled states of the qubits, while the oscillator will return to the ground state

$$
\psi\left(t>t_{3}\right)=|0\rangle\left(\cos \theta_{1} \mathrm{e}^{i \beta}|01\rangle+\sin \theta_{1} \mathrm{e}^{i \alpha}|10\rangle\right) .
$$

The manipulation should not necessarily be step-like; it is sufficient to pass the resonance rapidly enough to provide the Landau-Zener transition, i.e. the speed of the frequency ramping should be comparable to the qubit level splittings.

A somewhat more complex pulse sequence is required to realize a universal entangling two-qubit gate; the way to do it is explained, e.g., in Ref. 147.

\section{CONCLUSION AND PROSPECTIVES}

Within 5 years, engineered JJ quantum systems with 5-10 qubits will most likely begin seriously to test the scalability of solid state QI processors.

For this to happen, a few decisive initial steps and breakthroughs are needed and expected: The first essential step is to develop JJ-hardware with long coherence time to study the quantum dynamics of a two-qubit circuit and to perform a "test" of Bell's inequalities (or rather the JJ-circuitry) by creating entangled two-qubit Bell states and performing simultaneous projective measurements on the two qubits.

A first breakthrough would be to perform a significant number of single- and two-qubit gates on a 3-qubit cluster to entangle three qubits. Combined with simultaneous projective readout of individual qubits, not disturbing unmeasured qubits, this would form a basis for the first solid-state experiments with teleportation, quantum error correction (QEC), and elementary quantum algorithms. This will provide a platform for scaling up the system to 10 qubits.

This may not look very impressive but nevertheless would be an achievement far beyond expectations only a decade ago. The NMR successes, e.g., running Shor-type al- gorithms using a molecule with 7 qubits, ${ }^{148}$ are based on technologies developed during 50 years using natural systems with naturally long coherence times. Similarly, semiconductor technologies have developed for 50 years to reach today's scale and performance of classical computers. It is therefore to be expected that QI technologies will need several decades to develop truly significant potential. Moreover, in the same way as for the classical technologies, QI technologies will most probably develop slowly step by step, "qubit by qubit," which in itself will be an exponential development.

Moreover, in future scalable information processors, different physical realizations and technologies might be combined into hybrid systems to achieve fast processing in one system and long coherence and long-time information storage in another system. In this way, solid state technologies might be combined with ion trap physics to build large microtrap systems, ${ }^{149}$ which in turn might be coupled to superconducting Josephson junctions processors via microwave transmission lines. ${ }^{150}$

This work has been supported by the European Commission through the IST-SQUBIT and SQUBIT-2 projects, by the Swedish Research Council, the Swedish Foundation for Strategic Research, and the Royal Academy of Sciences.

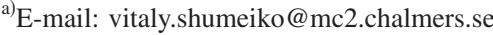

${ }^{1}$ R. P. Feynman, in Feynman Lectures on Computation, A. J. G. Hey and R. W. Allen (eds.), Reading, Massachusetts USA, Perseus Books (1996).

${ }^{2}$ M. A. Nielsen and I. L. Chuang, Quantum Computation and Quantum Information, Cambridge, UK, Cambridge University Press (2000).

${ }^{3}$ A. J. Legget, Suppl. Progr. Theor. Phys. 69, 80 (1980).

${ }^{4}$ A. J. Leggett, in Percolation, Localization, and Superconductivity, Goldman and Wolf (eds.) Plenum Press, New York (1984).

${ }^{5}$ A. J. Leggett and A. Garg, Phys. Rev. Lett. 54, 857 (1985).

${ }^{6}$ Y. Nakamura, Yu. Pashkin, and J. S. Tsai, Nature (London) 398, 786 (1999).

${ }^{7}$ A. O. Caldeira and A. Legget, Phys. Rev. Lett. 46, 211 (1981).

${ }^{8}$ M. H. Devoret, J. M. Martinis, and J. Clarke, Phys. Rev. Lett. 55, 1908 (1985).

${ }^{9}$ J. Clarke, A. N. Cleland, M. H. Devoret, D. Esteve, and J. M. Martinis, Science 239, 992 (1988).

${ }^{10}$ A. J. Leggett, S. Chakravarty, A. T. Dorsey, M. P. A. Fisher, A. Garg, and W. Zwerger, Rev. Mod. Phys. 59, 1 (1987).

${ }^{11}$ J. E. Mooij, T. P. Orlando, L. Levitov, Lin Tian, C. H. van der Waal, and S. Lloyd, Science 285, 1036 (1999).

${ }^{12}$ C. H. van der Waal, A. C. J. ter Haar, F. Wilhelm, R. N. Schouten, C. J. P. M. Harmans, T. P. Orlando, S. Lloydm, and J. E. Mooij, Science 290, 773 (2000).

${ }^{13}$ J. R. Friedman, V. Patel, W. Chen, S. K. Tolpygo, and J. E. Lukens, Nature (London) 406, 43 (2000).

${ }^{14}$ J. Martinis, S. Nam, J. Aumentado, and C. Urbina, Phys. Rev. Lett. 89, 1179011 (2002).

${ }^{15}$ J. M. Martinis, S. Nam, J. Aumentado, K. M. Lang, and C. Urbina, Phys. Rev. B 67, 094510 (2003).

${ }^{16}$ J. Claudon, F. Balestro, F. W. J. Hekking, and O. Buisson, Phys. Rev. Lett. 93, 187003 (2004).

${ }^{17}$ K. K. Likharev and A. Zorin, J. Low Temp. Phys. 59, 347 (1985).

${ }^{18}$ H. Grabert and M. H. Devoret (eds.), Single Charge Tunneling, NATO ASI Series, Plenum Press, New York (1992).

${ }^{19}$ P. Lafarge, P. Joyez, D. Esteve, C. Urbina, and M. H. Devoret, Nature (London) 365, 422 (1993)

${ }^{20}$ V. Bouchiat, P. Joyez, H. Pothier, C. Urbina, D. Esteve, and M. H. Devoret, Phys. Scr. T76, 165 (1998).

${ }^{21}$ A. Shnirman, G. Schön, and Z. Hermon, Phys. Rev. Lett. 79, 2371 (1997).

${ }^{22}$ G. Wendin, Philos. Trans. R. Soc. London, Ser. A 361, 1323 (2003). 
${ }^{23}$ G. Wendin, Phys. World, May (2003).

${ }^{24}$ M. H. Devoret and J. M. Martinis, Quantum Inform. Processing 3, 163 (2004).

${ }^{25}$ M. H. Devoret, A. Wallraff, and J. M. Martinis, "Superconducting Qubits: A Short Review," cond-mat/0411174 (2004).

${ }^{26}$ D. Esteve and D. Vion, Solid State Quantum Bit Circuits, Les Houches Summer School-Session LXXXI on Nanoscopic Quantum Physics (2004).

${ }^{27}$ D. Vion, A. Cottet, A. Aassime, P. Joyez, H. Pothier, C. Urbina, D. Esteve, and M. H. Devoret, Science 296, 886 (2002).

${ }^{28}$ D. Vion, A. Aassime, A. Cottet, P. Joyez, H. Pothier, C. Urbina, D. Esteve, and M. H. Devoret, Fortschritte Physik 51, 462 (2003).

${ }^{29}$ E. Collin, G. Ithier, A. Aassime, P. Joyez, D. Vion, and D. Esteve, Phys. Rev. Lett. 93, 157005 (2004).

${ }^{30}$ I. Chiorescu, Y. Nakamura, C. J. P. M. Harmans, and J. E. Mooij, Science 299, 1869 (2003).

${ }^{31}$ I. Chiorescu, P. Bertet, K. Semba, Y. Nakamura, C. J. P. M. Harmans, and J. E. Mooij, Nature (London) 431, 159 (2004).

${ }^{32}$ P. Bertet, I. Chiorescu, C. J. P. M. Harmans, J. E. Mooij, and K. Semba, Phys. Rev. B 70, 100501(R) (2004).

${ }^{33}$ E. Il'ichev, N. Oukhanski, A. Izmalkov, Th. Wagner, M. Grajcar, H.-G. Meyer, A. Yu. Smirnov, Alec Maassen van den Brink, M. H. S. Amin, and A. M. Zagoskin, Phys. Rev. Lett. 91, 0979061 (2003).

${ }^{34}$ T. Duty, D. Gunnarsson, K. Bladh, and P. Delsing, Phys. Rev. B 69, 1405023 (2004).

${ }^{35}$ R. W. Simmonds, K. M. Lang, D. A. Hite, D. P. Pappas, and John M. Martinis, Phys. Rev. Lett. 93, 077003 (2004).

${ }^{36}$ K. B. Cooper, M. Steffen, R. McDermott, R. W. Simmonds, S. Oh, D. A. Hite, D. P. Pappas, and John M. Martinis, Phys. Rev. Lett. 93, 180401 (2004).

${ }^{37}$ Yu. A. Pashkin, T. Yamamoto, O. Astafiev, Y. Nakamura, D. V. Averin, and J. S. Tsai, Nature (London) 421, 823 (2003).

${ }^{38}$ T. Yamamoto, Yu. Pashkin, O. Astafiev, Y. Nakamura, and J. S. Tsai, Nature (London) 425, 941 (2003)

${ }^{39}$ J. B. Majer, Superconducting Quantum Circuits, PhD thesis, TU Delft, The Netherlands (2002).

${ }^{40}$ J. B. Majer, J. B. Paauw, A. ter Haar, C. J. P. M. Harmans, and J. E. Mooij, Phys. Rev. Lett. 94, 090501 (2005).

${ }^{41}$ A. Izmalkov, M. Grajcar, E. Il'ichev, Th. Wagner, H.-G. Meyer, A. Y. Smirnov, M. H. S. Amin, Alec Maassen van den Brink, and A. M. Zagoskin, Phys. Rev. Lett. 93, 037003 (2004); A. Izmalkov, M. Grajcar, E. Il'ichev, Th. Wagner, H.-G. Meyer, A. Y. Smirnov, M. H. S. Amin, Alec Maassen van den Brink, and A. M. Zagoskin, Phys. Rev. Lett. 93 049902(E) (2004).

${ }^{42}$ A. J. Berkley, H. Xu, R. C. Ramos, M. A. Gubrud, F. W. Strach, P. R. Johnson, J. R. Anderson, A. J. Dagt, C. J. Lobb, and F. C. Wellstood, Science 368, 284 (2003).

${ }^{43}$ Yu. Makhlin, G. Schön, and A. Shnirman, Nature (London) 398, 305 (1999).

${ }^{44}$ Yu. Makhlin, G. Schön, and A. Shnirman, Rev. Mod. Phys. 73, 357 (2001).

${ }^{45}$ G. Burkard, in Handbook of Theoretical and Computational Nanotechnology, M. Rieth and W. Schommers (eds.), American Scientific Publishers, Los Angeles, (2006), Vol. 3; cond-mat/0409626.

${ }^{46} \mathrm{G}$. Wendin and V. S. Shumeiko, in Handbook of Theoretical and Computational Nanotechnology, M. Rieth and W. Schommers (eds.), American Scientific Publishers, Los Angeles (2006), Vol. 3, p. 223; cond-mat/ 0508729.

${ }^{47}$ K. K. Likharev, Dynamics of Josephson Junctions and Circuits, Gordon and Breach (1986).

${ }^{48}$ J. Weber, Phys. Rev. 90, 977 (1953).

${ }^{49}$ R. F. Voss and R. A. Webb, Phys. Rev. Lett. 47, 265 (1981).

${ }^{50}$ L. D. Landau and E. M. Lifshitz, Quantum Mechanics: Non-Relativistic Theory, Pergamon, Oxford (1977).

${ }^{51}$ J. M. Martinis, M. H. Devoret, and J. Clarke, Phys. Rev. B 35, 4682 (1987).

${ }^{52}$ B. Yurke and J. S. Denker, Phys. Rev. A 29, 1419 (1984).

${ }^{53}$ M. Devoret, in Quantum Fluctuations, Les Houches, Session LXIII, 1995, S. Reynaud, E. Giacobino, and J. Zinn-Justin (eds.), Elsevier (1997).

${ }^{54}$ G. Burkard, R. H. Koch, and D. P. DiVincenzo, Phys. Rev. B 69, 064503 (2004).

${ }^{55}$ M. A. Kastner, Rev. Mod. Phys. 64, 849 (1992).

${ }^{56}$ M. Büttiker, Phys. Rev. B 36, 3548 (1987).

${ }^{57}$ I. Giaever and H. R. Zeller, Phys. Rev. Lett. 20, 1504 (1968).

${ }^{58}$ I. O. Kulik and R. I. Shekhter, Sov. Phys. JETP 41, 308 (1975).

${ }^{59}$ P. Lafarge, P. Joyez, D. Esteve, C. Urbina, and M. H. Devoret, Phys. Rev. Lett. 70, 994 (1993).
${ }^{60}$ U. Weiss, Quantum Dissipative Systems, 2nd ed., World Scientific, Singapore (1999).

${ }^{61}$ Y. Nakamura, Yu. A. Pashkin, and J. S. Tsai, Phys. Rev. Lett. 87, 246601 (2002).

${ }^{62}$ Y. Nakamura, Yu. A. Pashkin, and J. S. Tsai, Phys. Rev. Lett. 88, 047901 (2002).

${ }^{63}$ Mahn-Soo Choi, R. Fazio, J. Siewert, and C. Bruder, Europhys. Lett. 53, 251 (2001).

${ }^{64}$ L. B. Ioffe, M. V. Feigel'man, A. Ioselevich, D. Ivanov, M. Troyer, and G. Blatter, Nature (London) 415, 503 (2002).

${ }^{65}$ M. V. Feigel'man, L. B. Ioffe, V. B. Geshkenbein, P. Dayal, and G. Blatter, Phys. Rev. Lett. 92, 098301 (2004).

${ }^{66}$ A. Zazunov, V. S. Shumeiko, E. Bratus, J. Lantz, and G. Wendin, Phys. Rev. Lett. 90, 0870031 (2003).

${ }^{67}$ A. Zazunov, V. S. Shumeiko, G. Wendin, and E. N. Bratus, Phys. Rev. B 71, 214505 (2005).

${ }^{68}$ A. Furusaki and M. Tsukada, Physica B 165-166, 967 (1990).

${ }^{69}$ V. S. Shumeiko, G. Wendin, and E. N. Bratus, Phys. Rev. B 48, 13129 (1993).

${ }^{70}$ V. S. Shumeiko, E. N. Bratus', and G. Wendin, "Dynamics of Andreev level qubits," in Electronic Correlations: from Meso- to Nano-physics, Proc. of XXXIII Moriond Conference, T. Martin, G. Montamboux, and J. T. Thanh Van (eds.), EDP Sciences (2001).

${ }^{71}$ J. Lantz, V. S. Shumeiko, E. N. Bratus', and G. Wendin, Physica C 368, 315 (2002).

${ }^{72}$ N. Chtchelkatchev and Y. Nazarov, Phys. Rev. Lett. 90, 226806 (2003).

${ }^{73}$ C. P. Slichter, Principles of Magnetic Resonance, Springer-Verlag, New York (1990)

${ }^{74}$ L. B. Ioffe, V. B. Geshkenbein, Ch. Helm, and G. Batter, Phys. Rev. Lett. 93, 057001 (2004).

${ }^{75}$ A. Shnirman, G. Schön, I. Martin, and Y. Makhlin, Phys. Rev. Lett. 94, 127002 (2005).

${ }^{76}$ J. M. Martinis et al., Phys. Rev. Lett. 95, 210503 (2005).

${ }^{77}$ K. Blum, Density Matrix: Theory and Applications, Plenum, New York (1996).

${ }^{78}$ Yu. Makhlin, G. Schön, and A. Shnirman, Phys. Rev. Lett. 85, 4578 (2000).

${ }^{79}$ G. Falci, E. Paladino, and R. Fazio, in Quantum Phenomena of Mesoscopic Systems, B. Altshuler and V. Tognetti (eds.), IOS Press, Amsterdam (2004); cond-mat/0312550.

${ }^{80}$ E. Paladino, L. Faoro, G. Falci, and Rosario Fazio, Phys. Rev. Lett. 88, 228304 (2002).

${ }^{81}$ E. Paladino, L. Faoro, and G. Falci, Adv. Solid State Phys. 43, 747 (2003).

${ }^{82}$ Yu. Makhlin and A. Shnirman, JETP Lett. 78, 497 (2003).

${ }^{83}$ Yu. Makhlin and A. Shnirman, Phys. Rev. Lett. 92, 178301 (2004).

${ }^{84}$ A. Shnirman, D. Mozyrsky, and I. Martin, Europhys. Lett. 67, 840 (2004).

${ }^{85}$ G. Falci, A. D'Arrigo, A. Mastellone, and E. Paladino, cond-mat/0409422.

${ }^{86}$ F. K. Wilhelm, G. Schön, and G. T. Zimanyi, Phys. Rev. Lett. 87, 136802 (2001).

${ }^{87}$ F. K. Wilhelm, M. J. Storcz, C. H. van der Waal, C. J. P. M. Harmans, and J. E. Mooij, Adv. Solid State Phys. 43, 763 (2003).

${ }^{88}$ M. C. Goorden and F. K. Wilhelm, Phys. Rev. B 68, 012508 (2003).

${ }^{89}$ C. H. van der Wal, F. K. Wilhelm, C. J. P. M. Harmans, and J. E. Mooij, Eur. Phys. J. B 31, 111 (2003).

${ }^{90}$ K. W. Lehnert, B. A. Turek, K. Bladh, L. F. Spietz, D. Gunnarsson, P. Delsing, and R. J. Schoelkopf, Phys. Rev. Lett. 91, 106801 (2003).

${ }^{91}$ K. W. Lehnert, K. Bladh, L. F. Spietz, D. Gunnarsson, D. I. Schuster, P. Delsing, and R. J. Schoelkopf, Phys. Rev. Lett. 90, 027002 (2003).

${ }^{92}$ L. Roschier, P. Hakonen, K. Bladh, P. Delsing, K. Lehnert, L. Spietz, and R. Schoelkopf, J. Appl. Phys. 95, 1274 (2004).

${ }^{93}$ G. Burkard, D. P. DiVincenzo, P. Bertet, I. Chiorescu, and J. E. Mooij, Phys. Rev. B 71, 134504 (2005).

${ }^{94}$ P. Bertet, I. Chiorescu, G. Burkard, K. Semba, C. J. P. M. Harmans, D. P. DiVincenzo, and J. E. Mooij, Phys. Rev. Lett. 95, 257002 (2005).

${ }^{95}$ D. V. Averin and R. Fazio, JETP Lett. 78, 1162 (2003).

${ }^{96}$ M. Governale, M. Grifoni, and G. Schön, Chem. Phys. 268, 273 (2001).

${ }^{97}$ M. J. Storcz and F. K. Wilhelm, Phys. Rev. A 67, 042319 (2003).

${ }^{98}$ K. Rabenstein, V. A. Sverdlov, and D. V. Averin, ZhETF Lett. 79, 783 (2004); cond-mat/0401519.

${ }^{99}$ K. Rabenstein and D. V. Averin, Turk. J. Phys 27, 1 (2003); cond-mat/ 0310193.

${ }^{100}$ M. Steffen et al., Phys. Rev. Lett. 97, 050502 (2006).

${ }^{101}$ Yu-Xi Liu, L. F. Wei, and F. Nori, Europhys. Lett. 67, 874 (2004).

${ }^{102}$ F. K. Wilhelm, Phys. Rev. 68, 060503(R) (2003).

${ }^{103}$ A. Aassime, G. Johansson, G. Wendin, R. J. Schoelkopf, and P. Delsing, 
Phys. Rev. Lett. 86, 3376 (2001).

${ }^{104}$ M. H. Devoret and R. J. Shoelkopf, Nature (London) 406, 1039 (2000).

${ }^{105}$ R. J. Schoelkopf, P. Wahlgren, A. A. Kozhevnikov, P. Delsing, and D. E. Prober, Science 280, 1238 (1998).

${ }^{106}$ A. Aassime, D. Gunnarsson, K. Bladh, R. S. Schoelkopf, and P. Delsing, Appl. Phys. Lett. 79, 4031 (2001).

${ }^{107}$ O. Astafiev, Yu. A. Pashkin, T. Yamamoto, Y. Nakamura, and J. S. Tsai, Phys. Rev. B 69, 180507(R) (2004).

${ }^{108}$ G. Johansson, A. Käck, and G. Wendin, Phys. Rev. Lett. 88, 046802 (2002).

${ }^{109}$ A. Käck, G. Johansson, and G. Wendin, Phys. Rev. B 67, 035301 (2003).

${ }^{110}$ D. V. Averin, Fortschritte Physik 48, 1055 (2000).

${ }^{111}$ A. N. Korotkov and D. V. Averin, Phys. Rev. B 64, 165310 (2001).

${ }^{112}$ D. V. Averin, Phys. Rev. Lett. 88, 207901 (2002).

${ }^{113}$ A. L. Shelankov and J. Rammer, Europhys. Lett. 63, 485 (2003).

${ }^{114}$ J. Rammer, A. L. Shelankov, and J. Wabnig, Phys. Rev. B 70, 115327 (2004).

${ }^{115}$ F. W. J. Hekking, O. Buisson, F. Balestro, and M. G. Vergniory, in Electronic Correlations: from Meso- to Nano-physics, Proc. of XXXIII Moriond Conference, T. Martin, G. Montamboux, and J. T. Thanh Van (eds.), EDP Sciences (2001), p. 515

${ }^{116}$ F. Marquardt and C. Bruder, Phys. Rev. 63, 054514 (2001).

${ }^{117}$ S. M. Girvin, Ren-Shou Huang, Alexandre Blais, Andreas Wallraff, and R. J. Schoelkopf, Prospects of Strong Cavity Quantum Electrodynamics with Superconducting Circuits, Proc. Les Houches Summer School, Session LXXIX, Quantum Entanglement and Information Processing (2003) cond-mat/0310670

${ }^{118}$ A. Blais, R.-S. Huang, A. Wallraff, S. M. Girvin, and R. J. Schoelkopf, Phys. Rev. A 69, 062320 (2004).

${ }^{119}$ I. Rau, G. Johansson, and A. Shnirman, Phys. Rev. B 70, 054521 (2004).

${ }^{120}$ A. Zorin, Physica C 368, 284 (2002).

${ }^{121}$ L. Roschier, M. Sillanpää, and P. Hakonen, Phys. Rev. B 71, 024530 (2005).

${ }^{122}$ A. Wallraff, D. Schuster, A. Blais, L. Frunzo, R. S. Huang, J. Majer, S. Kumar, S. M. Girvin, and R. J. Schoelkopf, Nature (London) 431, 165 (2004).

${ }^{123}$ D. I. Schuster, A. Wallraff, A. Blais, L. Frunzio, R. S. Huang, J. Majer, S. M. Girvin, and R. J. Schoelkopf, Phys. Rev. Lett. 94, 123602 (2005).

${ }^{124}$ A. Wallraff, D. Schuster, A. Blais, L. Frunzo, J. Majer, S. M. Girvin, and R. J. Schoelkopf, Phys. Rev. Lett. 95, 060501 (2005).

${ }^{125}$ G. Ithier, E. Collin, P. Joyez, P. Meeson, D. Vion, D. Esteve, F. Chiarello, A. Shnirman, Y. Makhlin, and G. Schön, Phys. Rev. B 72, 134519 (2005)

${ }^{126}$ T. Duty, G. Johansson, K. Bladh, D. Gunnarsson, C. Wilson, and P. Delsing, Phys. Rev. Lett. 95, 206807 (2005).

${ }^{127}$ G. Johansson, L. Tornberg, V. S. Shumeiko, and G. Wendin, J. Phys.: Condens. Matter 18, S901 (2006).
${ }^{128}$ G. Johansson, L. Tornberg, and C. M. Wilson, Phys. Rev. B 74, 100504(R) (2006).

${ }^{129}$ A. Cottet, D. Vion, P. Joyez, A. Aassime, D. Esteve, and M. H. Devoret, Physica C 367, 197 (2002).

${ }^{130}$ A. Lupascu, C. J. M. Verwijs, R. N. Schouten, C. J. P. M. Harmans, and J. E. Mooij, Phys. Rev. Lett. 93, 177006 (2004).

${ }^{131}$ I. Siddiqi, R. Vijay, F. Pierre, C. M. Wilson, L. Frunzio, M. Metcalfe, C. Rigetti, R. J. Schoelkopf, M. H. Devoret, D. Vion, and D. Esteve, Phys. Rev. Lett. 93, 207002 (2004).

${ }^{132}$ J. Siewert, R. Fazio, G. M. Palma, and E. Sciacca, J. Low Temp. Phys. 118, 795 (2000).

${ }^{133}$ J. Q. You, J. S. Tsai, and J. S. F. Nori, Phys. Rev. Lett. 89, 197902 (2002).

${ }^{134}$ J. Q. You, J. S. Tsai, and F. Nori, Phys. Rev. B 68, 024510 (2003).

${ }^{135}$ Y. D. Wang, P. Zhang, D. L. Zhou, and C. P. Sun, Phys. Rev. B 70, 224515 (2004).

${ }^{136}$ J. Lantz, M. Wallquist, V. S. Shumeiko, and G. Wendin, Phys. Rev. B 70, 140507 (2004).

${ }^{137}$ M. Wallquist, J. Lantz, V. S. Shumeiko, and G. Wendin, in Quantum Computation: Solid State Systems, P. Delsing, C. Granata, Y. Pashkin, B. Ruggiero, and P. Silvestrini (eds.), Kluwer Academic Plenum Publishers, December (2004).

${ }^{138}$ M. Wallquist, J. Lantz, V. S. Shumeiko, and G. Wendin, New J. Phys. 7, 178 (2005).

${ }^{139}$ L. F. Wei, Yu-Xi Liu, and F. Nori, Europhys. Lett. 67, 1004 (2004).

${ }^{140}$ C. Rigetti and M. Devoret, Phys. Rev. Lett. 94, 240502 (2005).

${ }^{141}$ M. J. Storcz and F. K. Wilhelm, Appl. Phys. Lett. 83, 2389 (2003).

${ }^{142}$ A. Blais, A. Maassen van den Brink, and A. M. Zagoskin, Phys. Rev. Lett. 90, 127901 (2003).

${ }^{143}$ D. V. Averin and C. Bruder, Phys. Rev. Lett. 91, 057003 (2003).

${ }^{144}$ F. W. Strauch, P. R. Johnson, A. J. Dragt, C. J. Lobb, J. R. Anderson, and F. C. Wellstood, Phys. Rev. Lett. 91, 167005 (2003).

${ }^{145}$ C. Cosmelli, M. G. Castellano, F. Chiarello, R. Leoni, G. Torrioli, and P. Carelli, cond-mat/0403690.

${ }^{146}$ M. Tinkham, Introduction to Superconductivity, McGraw Hill, New York (1996).

${ }^{147}$ M. Wallquist, V. S. Shumeiko, and G. Wendin, Phys. Rev. B 74, 224506 (2006).

${ }^{148}$ L. M. K. Vandersypen, M. Steffen, G. Breyta, C. S. Yannoni, M. H. Sherwood, and I. L. Chuang, Nature (London) 414, 883 (2001).

${ }^{149}$ A. M. Steane, quant-ph/0412165 (2004).

${ }^{150}$ L. Tian, P. Rabl, R. Blatt, and P. Zoller, Phys. Rev. Lett. 92, 247902 (2004).

This article was published in English in the original Russian journal. Reproduced here with stylistic changes by AIP. 